\title{
Thermoelectric transport through strongly correlated quantum dots
}

\author{
T. A. Costi $^{1}$ and V. Zlatic ${ }^{1,2}$ \\ ${ }^{1}$ Institut für Festkörperforschung, Forschungszentrum Jülich, 52425 Jülich, Germany \\ ${ }^{2}$ Institute of Physics, 10001 Zagreb, Croatia
}

(Received 31 March 2010; revised manuscript received 8 June 2010; published 24 June 2010)

\begin{abstract}
The thermoelectric properties of strongly correlated quantum dots, described by a single-level Anderson model coupled to conduction-electron leads, is investigated using Wilson's numerical renormalization-group method. We calculate the electronic contribution, $K_{\mathrm{e}}$, to the thermal conductance, the thermopower, $S$, and the electrical conductance, $G$, of a quantum dot as a function of both temperature, $T$, and gate voltage, $v_{g}$, for strong, intermediate, and weak Coulomb correlations, $U$, on the dot. For strong correlations and in the Kondo regime, we find that the thermopower exhibits two sign changes, at temperatures $T_{1}\left(v_{g}\right)$ and $T_{2}\left(v_{g}\right)$ with $T_{1}$ $<T_{2}$. We find that $T_{1}>T_{p}\left(v_{g}\right) \approx T_{K}\left(v_{g}\right)$, where $T_{p}\left(v_{g}\right)$ is the position of the Kondo-induced peak in the thermopower, $T_{K}\left(v_{g}\right)$ is the Kondo scale, and $T_{2}=O(\Gamma)$, where $\Gamma$ is the level width. The loci of $T_{1}\left(v_{g}\right)$ and $T_{2}\left(v_{g}\right)$ merge at a critical gate voltage, $v_{g}=v_{g}^{c}(U / \Gamma)$ beyond which no sign change occurs at finite gate voltage (measured relative to midvalley). We determine $v_{g}^{c}$ for different $U / \Gamma$ finding that $v_{g}^{c}$ coincides, in each case, with entry into the mixed-valence regime. No sign change is found outside the Kondo regime, or, for weak correlations $U / \Gamma \lesssim 1$, making such a sign change in $S(T)$ a particularly sensitive signature of strong correlations and Kondo physics. The relevance of this to recent thermopower measurements of Kondo correlated quantum dots is discussed. The results for quantum dots are compared also to the relevant transport coefficients of dilute magnetic impurities in nonmagnetic metals: the electronic contribution, $\kappa_{\mathrm{e}}$, to the thermal conductivity, the thermopower, $S$, and the impurity contribution to the electrical resistivity, $\rho$. In the mixed-valence and empty-orbital regimes, we find, as a function of temperature, two peaks in $K_{\mathrm{e}}$ as compared to a single peak in $\kappa_{\mathrm{e}}$, and similarly, $G(T)$ exhibits a finite-temperature peak on entering the mixed-valence regime whereas such a pronounced peak is absent in $\rho(T)$ even far into the empty-orbital regime. We compare and contrast the figure of merit, power factor, and the extent of violation of the Wiedemann-Franz law in quantum dots and dilute magnetic impurities. The extent of temperature scaling in the thermopower and thermal conductance of quantum dots in the Kondo regime is discussed.
\end{abstract}

DOI: 10.1103/PhysRevB.81.235127 PACS number(s): 72.10.Fk, 72.15.Qm, 72.15.Jf, 73.63.Kv

\section{INTRODUCTION}

Materials with potentially useful thermoelectric properties are currently under intense theoretical and experimental investigation, mainly due to the prospect of applications, e.g., for conversion of waste heat into electricity in thermoelectric generators, for applications to refrigeration or for on-chip cooling and energy efficiency in microelectronics applications. ${ }^{1-14}$ Apart from possible applications, thermoelectric materials can also serve as an interesting testing ground for theoretical approaches to electrical and thermal transport in solids. ${ }^{15-19}$ As the scale of the individual components in semiconducting devices is approaching the nanosize, a description of thermal transport through quantum dots is also attracting a lot of experimental and theoretical attention. ${ }^{20-22}$

In this paper, we address the thermoelectric properties of a nanoscale size quantum dot exhibiting the Kondo effect, which we describe in terms of a single-level Anderson impurity model with two conduction-electron leads at fixed chemical potentials. The quantum dots that we consider have sizes of 10-100 $\mathrm{nm}$ and can be tuned from the Kondo to the mixed-valence and empty-orbital regimes by a gate voltage. ${ }^{23-26}$ Short segments of carbon nanotubes ${ }^{27}$ connected to leads exhibit similar physics so our results could also be of relevance to such systems. Very recent experiments on nanoscale quantum dots ${ }^{20,28}$ are beginning to probe the effect of Kondo correlations on the thermopower, although as we shall argue in the conclusions a quantitative comparison with theory is still some way off. The thermoelectric properties of dilute magnetic impurities in nonmagnetic metals, such as $\mathrm{Ce}_{x} \mathrm{La}_{1-x} \mathrm{Al}_{3}$ and $\mathrm{Ce}_{x} \mathrm{La}_{1-x} \mathrm{~B}_{6}$, are closely related to those of quantum dots (see Sec. II) so we discuss these here also. Understanding the thermoelectric properties of magnetic impurities is also a useful starting point for understanding those of heavy fermions within the dynamical mean-field theory approach ${ }^{19}$ although, in these systems, crystal-field effects and nonresonant channels play a crucial role for the thermopower, and need to be taken into account for a quantitative comparison to experiment. ${ }^{16-18}$

The approach that we use in this paper, Wilson's numerical renormalization-group (NRG) method, ${ }^{29-31}$ gives reliable results for transport properties in all parameter and temperature regimes of interest. ${ }^{15}$ The present calculations were carried out for the Anderson model with finite Coulomb repulsion, as is appropriate for nanoscale size quantum dots. We implemented recent developments in the calculation of dynamical quantities within the NRG, including the use of the self-energy $^{32}$ and the full density-matrix (FDM) generalization $^{33}$ (see also Refs. 34 and 35) of the reduced density-matrix approach ${ }^{36}$ within the complete basis set of eliminated states. ${ }^{37}$ In particular, the FDM approach allows calculations of dynamical properties at all excitation energies $\omega$ relative to the temperature $T$, thereby simplifying the calculation of transport properties which require knowledge of 
excitations, $\omega$, above and below the temperature. ${ }^{15}$

The outline of the paper is as follows. In Secs. II and III, we describe the Anderson impurity model for quantum dots and dilute magnetic impurities and we specify the relevant transport quantities that we calculate for these two different physical realizations of the model. The NRG method used in this paper is described in Sec. IV together with results for occupancies which we use to define Kondo, mixed-valence, and empty-orbital regimes in the strong correlation limit. Section V presents the temperature-dependent transport properties of quantum dots and Sec. VI compares these to the corresponding quantities for dilute magnetic impurities. Results for the figure of merit, power factor, and Lorenz number ratios for quantum dot and magnetic impurity systems are presented in Sec. VII. Section VIII investigates the extent to which universal scaling functions apply to the thermopower and thermal conductance of quantum dots in the Kondo regime. In Sec. IX, we present our results for the gate-voltage (local level) dependence of transport quantities for quantum dots (magnetic impurities). Conclusions and a discussion of the relevance of our results to recent experiments on nanoscale size quantum dots is presented in Sec. X. Appendix A discusses the reduction of the two-lead Anderson model to a single-channel model, Appendix B contains some additional results for moderately and weakly correlated quantum dots, and Appendix C provides details of the FDM approach $^{33}$ and an alternative detailed derivation of the FDM expression for local Green's functions, which we have used to obtain the results in this paper. Finally Appendix D gives an outline of the derivation of thermopower and thermal conductance for quantum dots.

\section{MODEL}

A nanoscale quantum dot is described by the single-level Anderson impurity model with two conduction-electron leads,

$$
\begin{aligned}
H= & \sum_{\alpha k \sigma} \epsilon_{\alpha k \sigma} c_{\alpha k \sigma}^{\dagger} c_{\alpha k \sigma}+\sum_{\sigma} \varepsilon_{d} d_{\sigma}^{\dagger} d_{\sigma}+U n_{d \uparrow} n_{d \downarrow} \\
& +\sum_{\alpha k \sigma} t_{\alpha}\left(c_{\alpha k \sigma}^{\dagger} d_{\sigma}+\text { H.c. }\right) .
\end{aligned}
$$

Here, $\epsilon_{\alpha k \sigma}$ is the kinetic energy of conduction electrons with wave number $k$ and spin $\sigma$ in lead $\alpha=(L, R), \varepsilon_{d}$ is the local level energy, $U$ is the Coulomb repulsion on the dot, and $t_{\alpha}$ is the tunnel matrix element of the dot level to conductionelectron states in lead $\alpha=(L, R)$. The operators $c_{\alpha k \sigma}^{\dagger}\left(c_{\alpha k \sigma}\right)$ create (destroy) conduction-electron states $|\alpha k \sigma\rangle$ and $d_{\sigma}^{\dagger}\left(d_{\sigma}\right)$ create (destroy) local $d$-level states $|\sigma\rangle$. We assume a flat density of states of magnitude $N_{F}=1 / 2 D$ per spin channel for both leads, where $D=1$ is the half bandwidth of each lead. The single-particle broadening (half width at half maximum) of the $d$ level is given by $\widetilde{\Gamma}=\widetilde{\Gamma}_{L}+\widetilde{\Gamma}_{R}$, where $\widetilde{\Gamma}_{L, R}$ $=\pi N_{F} t_{L, R}^{2}$ are the contributions to the broadening from the left and right leads. In this paper, we follow the convention used in quantum dot work and use as unit of energy not $\widetilde{\Gamma}$ but the full width at half maximum $\Gamma=2 \widetilde{\Gamma}$.
Since the $d$ state of the quantum dot in Eq. (1) only couples to the even combination $a_{e k \sigma} \sim t_{L} c_{L k \sigma}+t_{R} c_{R k \sigma}$ of the lead electron states, one can show (see Appendix A) that, to a very good approximation, the above model can be reduced to the following single-channel Anderson model:

$$
\begin{aligned}
H= & \sum_{k \sigma} \epsilon_{e k \sigma} a_{e k \sigma}^{\dagger} a_{e k \sigma}+\sum_{\sigma} \varepsilon_{d} d_{\sigma}^{\dagger} d_{\sigma}+U n_{d \uparrow} n_{d \downarrow} \\
& +t \sum_{k \sigma}\left(a_{e k \sigma}^{\dagger} d_{\sigma}+\text { H.c. }\right),
\end{aligned}
$$

where the tunneling amplitude $t$ is given by $t^{2}=t_{L}^{2}+t_{R}^{2}$ so that the hybridization strength of the dot to the leads is given by $\widetilde{\Gamma}=\widetilde{\Gamma}_{L}+\widetilde{\Gamma}_{R}$. This is also the appropriate model for describing dilute magnetic impurities in nonmagnetic metals. ${ }^{15}$ In fact, for both systems (see below and Appendix A), the calculation of the linear transport properties reduces to the calculation of the equilibrium $d$-level spectral density of the singlechannel model,

$$
A(\omega)=-\frac{1}{\pi} \operatorname{Im}\left[G_{d \sigma}(\omega+i \delta)\right],
$$

where $G_{d \sigma}(\omega+i \delta)=\left\langle\left\langle d_{\sigma} ; d_{\sigma}^{\dagger}\right\rangle\right\rangle$ is the Fourier transform of the retarded $d$-level Green's function of Eq. (2). Hence, all results in this paper, including those for dilute magnetic impurities, are obtained by solving the single-channel model (2) using the NRG (as explained in Sec. IV) to obtain $A(\omega, T)$.

\section{TRANSPORT QUANTITIES}

\section{A. Quantum dots}

Thermoelectric transport through the quantum dot [Eq. (1)] is calculated for a steady-state situation in which a small external bias voltage, $\delta V=V_{L}-V_{R}$, and a small temperature gradient $\delta T$ is applied between the left and right leads. Left and right leads are then at different chemical potentials $\mu_{L}$ and $\mu_{R}$, and temperatures $T_{L}$ and $T_{R}$, with $e \delta V=\mu_{L}-\mu_{R}$ and $\delta T=T_{L}-T_{R}$. We follow the approach for deriving the electrical conductance, $G(T)$, the thermal conductance, $K_{\mathrm{e}}(T)$, and thermoelectric power, $S(T)$, through an interacting quantum $\operatorname{dot}^{38-40}$ using the nonequilibrium Green's-function formalism. For completeness, an outline of this derivation ${ }^{21,22}$ can be found in Appendix D. The final expressions are given by

$$
\begin{gathered}
G(T)=e^{2} I_{0}(T), \\
S(T)=-\frac{1}{|e| T} \frac{I_{1}(T)}{I_{0}(T)}, \\
K_{\mathrm{e}}(T)=\frac{1}{T}\left[I_{2}(T)-\frac{I_{1}^{2}(T)}{I_{0}(T)}\right],
\end{gathered}
$$

where $I_{n}, n=0,1,2$ are the transport integrals

$$
I_{n}(T)=\frac{2}{h} \int d \omega \omega^{n} \mathcal{T}(\omega)\left(-\frac{\partial f}{\partial \omega}\right) .
$$

Here, $e$ denotes the magnitude of the electronic charge and $h$ denotes Planck's constant. The quantity $\mathcal{T}(\omega)$ is related to the spectral density $A(\omega)$ via 


$$
\mathcal{T}(\omega)=4 \pi \frac{\widetilde{\Gamma}_{L} \widetilde{\Gamma}_{R}}{\widetilde{\Gamma}_{L}+\widetilde{\Gamma}_{R}} A(\omega) .
$$

At $T=0$, the conductance acquires the value

$$
\begin{aligned}
G(0) & =\frac{2 e^{2}}{h} \mathcal{T}(0) \\
& =\frac{2 e^{2}}{h} \frac{4 \widetilde{\Gamma}_{L} \widetilde{\Gamma}_{R}}{\left(\widetilde{\Gamma}_{L}+\widetilde{\Gamma}_{R}\right)^{2}} \sin ^{2}\left(\pi n_{d} / 2\right),
\end{aligned}
$$

where $n_{d}$ is the occupancy of the dot and we have used the Friedel sum rule,

$$
A(\omega=0, T=0)=\frac{1}{\pi\left(\widetilde{\Gamma}_{L}+\widetilde{\Gamma}_{R}\right)} \sin ^{2}\left(\pi n_{d} / 2\right) .
$$

For integer occupation, $n_{d}=1$, and equal coupling to the leads, $\widetilde{\Gamma}_{L}=\widetilde{\Gamma}_{R}$, the conductance reaches the unitary value $2 e^{2} / h$, which we henceforth denote by $G_{0}$.

\section{B. Dilute magnetic impurities}

It is of interest to compare the transport properties of a quantum dot, with the corresponding quantities for electrons scattering from a dilute concentration, $n_{i} \ll 1$, of magnetic impurities in a clean host metal with constant density of states $N_{F}$ per spin. As for quantum dots, the relevant model for such dilute magnetic impurities is the single-channel Anderson model (2) with hybridization strength $\widetilde{\Gamma}$. In order to obtain the thermopower, $S(T)$, the thermal conductivity, $\kappa_{\mathrm{e}}(T)$, and resistivity, $\rho(T)$ (or conductivity $\sigma=1 / \rho$ ) for such a dilute concentration of magnetic impurities we use the Kubo formalism, see Appendix A of Ref. 15 for the details, and find for these quantities,

$$
\begin{gathered}
\rho(T)=\frac{1}{e^{2} M_{0}(T)}, \\
S(T)=-\frac{1}{|e| T} \frac{M_{1}(T)}{M_{0}(T)}, \\
\kappa_{\mathrm{e}}(T)=\frac{1}{T}\left[M_{2}(T)-\frac{M_{1}^{2}(T)}{M_{0}(T)}\right] .
\end{gathered}
$$

The transport integrals $M_{n}, n=0,1,2$, appearing here, are now defined by

$$
M_{n}(T)=\int d \omega \omega^{n} \tau(\omega, T)\left(-\frac{\partial f}{\partial \omega}\right),
$$

where $\tau(\omega, T)$ is the transport time of electrons, which is given in terms of the impurity spectral density $A(\omega, T)$ by

$$
\frac{1}{\tau(\omega, T)}=\frac{n_{i}}{N_{F}} 2 \widetilde{\Gamma} A(\omega, T) .
$$

In order to compare impurity transport properties with those of quantum dots, using the same units, we shall use rescaled quantities, e.g., for quantum dots $G / G_{0}$ and $K_{\mathrm{e}} / G_{0}$ and for impurities $\rho / \rho_{0}$ and $\kappa_{\mathrm{e}} \rho_{0}$ where

$$
\rho_{0}=2 n_{i} / \pi N_{F} e^{2}
$$

is the unitary resistivity of electrons scattering from a dilute concentration $n_{i}$ of magnetic impurities.

While the physics governing the transport properties of electrons scattering from dilute magnetic impurities, described by Eq. (2), is expected to be similar to that governing the transport properties of electrons through quantum dots [also described by Eq. (2)], differences are also expected, particularly for the respective thermopowers or the thermal conductance (conductivity), for the following reason: the transport expressions for quantum dots arise from integrals $I_{n}, n=0,1,2$ which involve the $n$th moments of $A(\omega, T)$ convoluted with the derivative of a Fermi function whereas those for magnetic impurities arise from $n$th moments of $1 / A(\omega, T)$ convoluted with the same derivative. At low temperatures, a Sommerfeld expansion for $I_{1}$ and $M_{1}$ results in different signs for the thermopower in the two different situations since derivatives of $A$ and $1 / A$ have opposite signs. On the other hand, at higher temperatures, moments of $A$ and $1 / A$ are determining factors for transport. Particularly the moments $I_{1}\left(M_{1}\right)$, entering the thermopower, and $I_{2}\left(M_{2}\right)$, entering the thermal conductance (conductivity), probe differences in the behavior or $A(\omega, T)$ and $1 / A(\omega, T)$ at high temperature. Consequently we expect significant quantitative differences for the thermopower and thermal conductance (conductivity) of quantum dots and dilute magnetic impurities at high temperatures. We discuss these differences in Sec. VI.

\section{NRG APPROACH}

\section{A. NRG and dynamical quantities}

We calculate the spectral function $A(\omega, T)$ and the transport properties of quantum dots, by using the NRG approach. ${ }^{29-31}$ This method is numerically exact and can be used to calculate both static thermodynamic properties as well as finite-temperature dynamic and transport properties. ${ }^{15}$ In brief, the NRG procedure ${ }^{29,30}$ consists of the following steps, (i), a logarithmic mesh of $\varepsilon_{k}^{n}=D \Lambda^{-n}$ is introduced about the Fermi level $\varepsilon_{F}=0$, and, (ii), a unitary transformation of the $a_{e k \sigma}$ in Eq. (2) is performed such that $f_{0 \sigma}$ $=\sum_{k} a_{e k \sigma}$ is the first operator in a new basis, $f_{n \sigma}, n=0,1, \ldots$, which tridiagonalizes $H_{c}=\Sigma_{k \sigma} \epsilon_{e k \sigma} a_{e k \sigma}^{\dagger} a_{e k \sigma}$, i.e., $H_{c}$ $\rightarrow \Sigma_{\sigma} \Sigma_{n=0}^{\infty} t_{n}\left(f_{n+1 \sigma}^{\dagger} f_{n \sigma}+\right.$ H.c. $)$, where the hoppings $t_{n} \approx \Lambda^{-n / 2}$ for a flat conduction band. ${ }^{30}$ The Hamiltonian (2) with the above discretized form of the kinetic energy is now iteratively diagonalized by defining a sequence of finite-size Hamiltonians,

$$
\begin{aligned}
H_{m}= & \sum_{\sigma} \varepsilon_{d} d_{\sigma}^{\dagger} d_{\sigma}+U n_{d \uparrow} n_{d \downarrow}+t \sum_{\sigma}\left(f_{0 \sigma}^{\dagger} d_{\sigma}+\text { H.c. }\right) \\
& +\sum_{n=0, \sigma}^{m-1} t_{n}\left(f_{n+1 \sigma}^{\dagger} f_{n \sigma}+\text { H.c. }\right)
\end{aligned}
$$

for $m \geq 0$ up to a maximum chain length $N$. For each $m$, this 
yields the excitations $E_{p}^{m}$ and many-body eigenstates $|p m\rangle$ of $H_{m}$ at a corresponding set of energy scales $\omega_{m}$ defined by the smallest scale in $H_{m}, \omega_{m}=t_{m} \approx \Lambda^{-(m-1) / 2}$. Since the number of states grows as $4^{m}$, for $m>6$ only the lowest 600 or so states of $H_{m}$ are retained. These are used as a basis for constructing $H_{m+1}$. For $m>6$, both the retained and eliminated (highenergy) states of $H_{m}$, together with the corresponding eigenvalues, are stored. This information is subsequently used to evaluate the spectral function $A(\omega, T)$ within the FDM approach $^{33}$ described in Appendix C. This evaluation makes use of, (i), the completeness of eliminated states, ${ }^{37}$ allowing a multiple-shell evaluation of Green's functions, ${ }^{41}$ avoiding double counting of excitations, and, (ii), the reduced densitymatrix approach to Green's functions, introduced to the NRG by Hofstetter. ${ }^{36}$ In addition, we calculate the spectral function via the correlation part of the self-energy $\Sigma(\omega, T)$ following Bulla et al., ${ }^{32}$ via

$$
\begin{aligned}
& A(\omega, T)=-\frac{1}{\pi} \operatorname{Im}\left[\frac{1}{\omega-\epsilon_{d}+i \Gamma-\Sigma(\omega, T)}\right], \\
& \Sigma(\omega, T)=U \frac{\left\langle\left\langle n_{d,-\sigma} d_{\sigma} ; d_{\sigma}^{\dagger}\right\rangle\right\rangle}{\left\langle\left\langle d_{\sigma} ; d_{\sigma}^{\dagger}\right\rangle\right\rangle} \equiv U \frac{F_{\sigma}(\omega, T)}{G_{d \sigma}(\omega, T)} .
\end{aligned}
$$

Since the FDM entering the definition of the Green's functions, see Appendix C, contains the complete spectrum from all NRG iterations, asymptotically high and low temperatures can be investigated more easily than within previous approaches ${ }^{15}$ which involved at a given temperature $T$, choosing an appropriate energy shell to extract $A(\omega, T)$. In addition, the regime $\omega \ll T$, which was problematical in previous approaches, can now be addressed, since contributions from all excitations (for all energy shells) are taken into account in the expression for the Green's function within the FDM approach.

\section{B. Calculations}

The calculations reported here have been carried out for a discretization parameter $\Lambda=1.75$, retaining 660 states per NRG iteration and a hybridization strength $\widetilde{\Gamma}=0.01$ (in units of the half bandwidth $D=1$ ). The maximum chain length diagonalized was $N=68$. We use the full width $\Gamma=2 \widetilde{\Gamma}=0.02$ as our energy unit throughout. Results for a wide range of temperatures from $T / \Gamma \ll 1$ to $T / \Gamma \gg 1$ were obtained to fully characterize the transport properties of quantum dots and dilute magnetic impurities. We note that, in practice, the regime $T / \Gamma \gg 1$ is probably not accessible in experiment due to other effects which become important at high temperature, and which we do not take into account, e.g., phonons, multiple levels, crystal-field states, etc. Calculations for strong $(U / \Gamma=8,6)$, moderate $(U / \Gamma=3)$, and weak $(U / \Gamma=1)$ correlations were carried out for a range of dimensionless gate voltages, $v_{g}$, defined by

$$
v_{g}=\frac{\varepsilon_{d}+U / 2}{\Gamma}=0.25 n, \quad n= \pm 1, \pm 2, \ldots, \pm 32 .
$$

With this definition, the gate voltage for midvalley occurs at $v_{g}=0$ for all $U$. Due to particle-hole symmetry, calculations

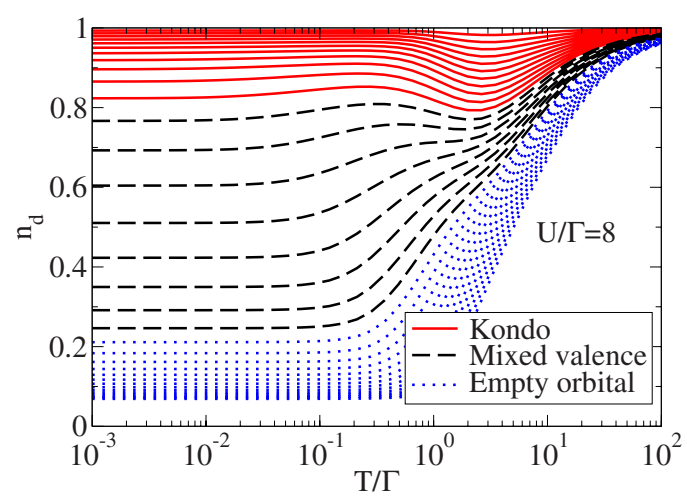

FIG. 1. (Color online) Temperature dependence of the occupancy for different values of the gate voltages $v_{g}>0$ in the Kondo (solid lines), mixed-valence (dashed lines), and empty-orbital (dotted lines) regimes. We define these regimes by $\left|n_{d}(T=0)-1\right|$ $\lesssim 0.25,\left|n_{d}(T=0)-0.50\right| \lesssim 0.25$, and $\left|n_{d}(T=0)\right| \lesssim 0.25$, respectively.

were carried out for $v_{g}>0$, with those for $v_{g}<0$ being obtained via a particle-hole transformation. This results in $v_{g}$ $\rightarrow-v_{g}$, occupancy $n_{d} \rightarrow 1-n_{d}$, double occupancy $D$ $=\left\langle n_{d \uparrow} n_{d \downarrow}\right\rangle \rightarrow 1-n_{d}+D$, thermopower $S \rightarrow-S$, with $G$ and $K_{\mathrm{e}}$ remaining unchanged. The behavior of $S, G$, and $K_{\mathrm{e}}$ under $v_{g} \rightarrow-v_{g}$, follows from their definition and the behavior of the spectral function, $A(\omega, T) \rightarrow A(-\omega, T)$ under $v_{g} \rightarrow-v_{g}$.

Figure 1 shows the temperature dependence of the dotlevel occupancy, $n_{d}(T)$, for gate voltages in the Kondo, mixed-valence, and empty-orbital regimes, for $U / \Gamma=8$. For $U / \Gamma \gg 1$, we use the occupancy at $T=0$ to delineate between the different regimes. Specifically, the Kondo regime is defined by gate voltages around midvalley $\left(v_{g}=0\right)$ with $\mid n_{d}(T$ $=0)-1 \mid \lesssim 0.25$ (see caption of Fig. 1). Similarly, the mixedvalence and empty-orbital regimes are defined by gate voltages corresponding to $\left|n_{d}(T=0)-0.5\right| \lesssim 0.25$ and $n_{d}(T=0)$ $<0.25$, respectively (see Fig. 1). In the Kondo regime, a characteristic low-temperature scale, the Kondo scale $T_{K}$, can be defined via ${ }^{42}$

$$
T_{K}=U \sqrt{\frac{\tilde{\Gamma}}{2 U}} e^{\pi \epsilon_{d}\left(\epsilon_{d}+U\right) / 2 \tilde{\Gamma} U}=\Gamma \sqrt{\frac{\widetilde{u}}{4}} e^{\pi\left(v_{g}^{2}-\tilde{u}^{2} / 4\right) / \widetilde{u}},
$$

where $\widetilde{u}=U / \Gamma$. The midvalley Kondo scales for $\widetilde{u}=3,6$, and 8 , are $T_{K} / \Gamma=8.2 \times 10^{-2}, 1.0 \times 10^{-2}$, and $2.64 \times 10^{-3}$, respectively.

Within the FDM approach, the thermodynamic value of the dot occupancy $n_{d}=\operatorname{Tr}\left[\rho n_{d}\right]$, where $\rho$ is the FDM defined in Appendix $\mathrm{C}$, and the value obtained from the spectral sum rule $n_{d}=\Sigma_{\sigma} \int_{-\infty}^{+\infty}-\frac{1}{\pi} \operatorname{Im}\left[F_{\sigma}(\omega, T)\right] d \omega$, with $F_{\sigma}$ defined in Eq. (18), are identical by construction, as we also verified numerically. Figure 2 shows the gate-voltage dependence of the dot occupancy (and for completeness also the double occupancy $\left.D=\left\langle n_{d \uparrow} n_{d \downarrow}\right\rangle\right)$ at a number of temperatures for the strong correlation case $U / \Gamma=8$.

\section{Physics of Kondo, mixed-valence, and empty-orbital regimes}

Before presenting the results, a few words are in order concerning the physical significance of the Kondo, mixed- 


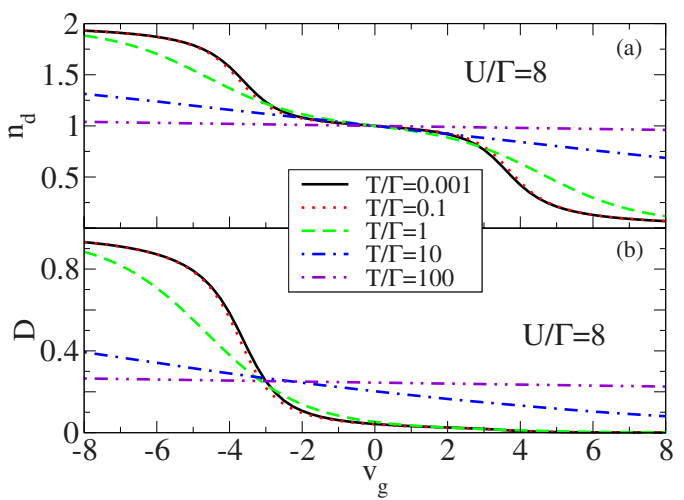

FIG. 2. (Color online) Dependence on gate voltage, $v_{g}=\left(\varepsilon_{d}\right.$ $+U / 2) / \Gamma$, of, (a), the occupancy of the quantum dot, $n_{d}$, and, (b), the double occupancy, $D=\left\langle n_{d \uparrow} n_{d \downarrow}\right\rangle$, at several temperatures.

valence, and empty-orbital regimes for strong Coulomb correlations $(U / \Gamma \gg 1)$ on the dot (for more detailed information we refer the reader to Ref. 42 ). The Kondo regime, $n_{d} \approx 1$, corresponds to the formation of a localized spin on the dot at intermediate temperatures $\left(T_{K} \leq T \ll \Gamma\right)$. In this temperature range, physical properties exhibit logarithmic temperature dependences, the hallmark of the Kondo effect. At $T<T_{K}$, the localized spin is quenched by the lead electrons, resulting in a many-body singlet at $T=0$ and a narrow Kondo resonance (of width $T_{K}$ ) in the dot spectral density at the Fermi level. Physical properties are characterized by spin fluctuations on scales $T_{K} \leq T \ll \Gamma$, charge fluctuations on a scale $T$ $\approx \Gamma$, and renormalized Fermi-liquid excitations at $T \ll T_{K}$. The dot spectral density is well understood: ${ }^{15}$ it has a threepeaked structure, with single-particle charge excitations at $\varepsilon_{d}$ and $\varepsilon_{d}+U$ and a temperature-dependent Kondo resonance at the Fermi level. The mixed-valence regime, corresponds to gate voltages such that the level $\varepsilon_{d}$ is within $\widetilde{\Gamma}$ of the Fermi level. The charge on the dot fluctuates between $n_{d}=0$ and $n_{d}=1$ resulting in an average charge $n_{d} \approx 0.5$. The physics is governed by quantum-mechanical charge fluctuations on a scale set by $\widetilde{\Gamma}$. The empty-orbital regime, corresponds to $n_{d}$ $\approx 0$ and $\varepsilon_{d} / \Gamma \gg 1$. Physical properties are dominated by charge fluctuations, primarily via thermal activation (with an activation energy $\varepsilon_{d}$ ). Even though $U / \Gamma \gg 1$, the physics in this regime corresponds to that of a noninteracting resonantlevel model with a resonant level of width $\widetilde{\Gamma}$ at energy $\varepsilon_{d}$ $>0$.

\section{TEMPERATURE DEPENDENCE OF TRANSPORT PROPERTIES OF QUANTUM DOTS}

The temperature dependence of transport properties of a quantum dot described by the model (1) is shown in Fig. 3 for several values of the gate voltage, ranging from the Kondo regime [Figs. 3(a), 3(d), and 3(g)], to the mixedvalence [Figs. 3(b), 3(e), and 3(h)] and empty orbital [Figs. 3(c), 3(f), and 3(i)] regimes and for strong Coulomb correlations on the dot $(U / \Gamma=8)$. Moderate to weak correlations are described briefly in Sec. V D and Appendix B. Depending on the regime, the transport properties exhibit different charac-
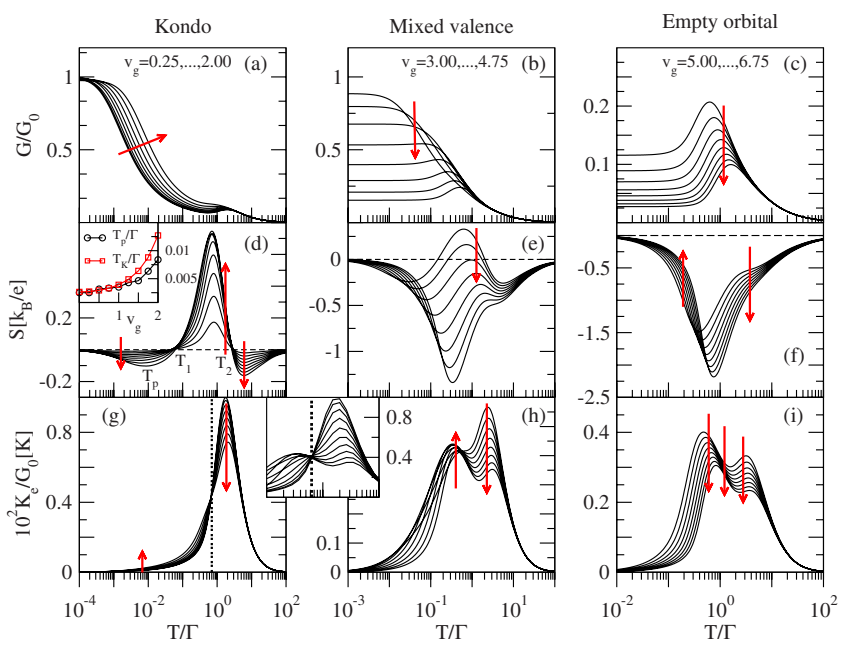

FIG. 3. (Color online) (a)-(c) The normalized electrical conductance, $G / G_{0}$, where $G_{0}=2 e^{2} / h$, (d)-(f), the thermopower, $S$, in units of $k_{B} / e$, and, (g)-(i), the normalized electronic contribution to the thermal conductance, $K_{\mathrm{e}} / G_{0}$, multiplied by a factor $10^{2}$ for clarity of presentation, as a function of $T / \Gamma$, in the strongly correlated regime $U / \Gamma=8$ for a range of gate voltages, $v_{g}=\left(\varepsilon_{d}+U / 2\right) / \Gamma$, in the Kondo (first column), mixed-valence (second column), and empty-orbital (third column) regimes. The range of $v_{g}$ is indicated in the top panels for each regime and the increment used was 0.25 . Arrows indicate the evolution of the transport quantities with increasing $v_{g}$. The inset of (d) compares the Kondo scale $T_{K}$ in the Kondo regime with the peak position of the low-temperature peak, $T_{p}$, in $S$ below the first sign change at $T_{1}$ as a function of $v_{g}>0$. In the Kondo regime $T_{K} \approx T_{p}$ and for gate voltages approaching the mixed-valence regime, the two scales deviate, as expected. The inset for $K_{\mathrm{e}}$ in $(\mathrm{g})$ and $(\mathrm{h})$ shows the crossing point at $T / \Gamma \approx 0.6$ in more detail (vertical dotted line) and the evolution of the twopeaked structure for gate voltages approaching the mixed-valence regime.

teristic temperature dependences, which we describe in detail below for each transport property in turn. Here, and in several other figures in the paper, we use arrows to indicate the evolution, with increasing gate voltage $v_{g}>0$ about midvalley $\left(v_{g}=0\right)$, of the various transport properties.

\section{A. Electrical conductance: $G(T)$}

The general trends in the electrical conductance $G(T)$ of Kondo correlated quantum dots are well understood: $:^{15,43-47}$ in short, as $T \rightarrow 0$, the conductance approaches a maximum value [see Fig. 3(a)], indicating that the quantum dot appears "transparent" to electrons tunneling through it, and a logarithmic behavior around $T_{K}$ marks the crossover from the weakly coupled regime at $T \gg T_{K}$ to the strongly coupled regime at $T \ll T_{K}$. An issue, less discussed in the literature, which we point out here, is the appearance of a finitetemperature peak in the conductance, $G(T) / G_{0}$, on entering the mixed-valence regime [see Fig. 3(b)]. This feature becomes particularly pronounced in the empty-orbital regime [see Fig. 3(c)]. This effect has been observed in experiments on lateral quantum $\operatorname{dots}^{23,26}$ and a comparison to theoretical calculations shows good agreement ${ }^{48,49}$ (see also the discus- 


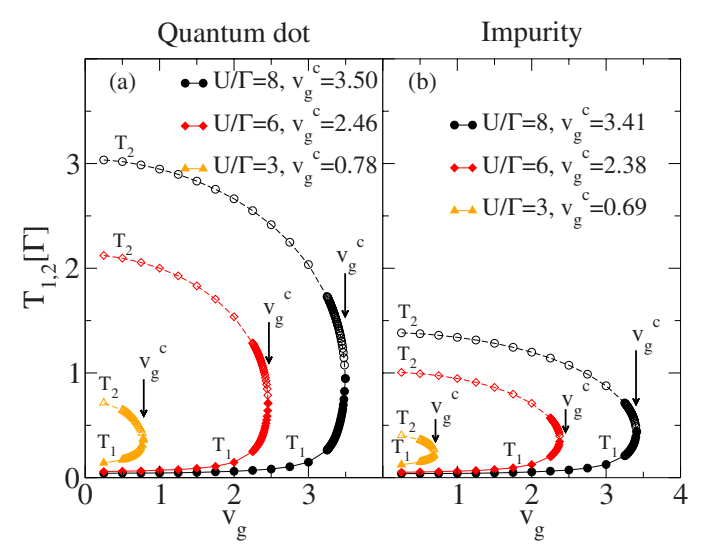

FIG. 4. (Color online) Dependence of the temperatures $T_{1}$ (filled symbols, solid lines) and $T_{2}$ (open symbols, dashed lines) at which $S(T)$ changes sign as a function of $v_{g} \geq 0.25$ for $U / \Gamma=3,6,8$ (left panel: quantum dot, right panel: magnetic impurity). The critical gate voltage, $v_{g}^{c}$, beyond which no sign change occurs in $S(T)$ at finite $v_{g}$ is indicated in the legend for each case.

sion of the resistivity of dilute magnetic impurities in Sec. VI).

\section{B. Thermopower: $S(T)$}

The thermopower exhibits a particularly interesting temperature dependence in the Kondo regime, Fig. 3(d), with two sign changes at $T=T_{1}\left(v_{g}\right)$ and $T=T_{2}\left(v_{g}\right)$, and, correspondingly, three extrema at $T=T_{p}, T \approx 0.6 \Gamma-0.8 \Gamma$, and $T$ $\approx 6 \Gamma$. The detailed behavior of $T_{1}$ and $T_{2}$ as a function of gate voltage will be described below; here, it suffices to note that neither $T_{1}$ nor $T_{2}$ are low-energy scales, and $T_{2}$ is typically on a scale of order $\Gamma$ [see Figs. 3(d) and 4(a) below]. The low-temperature "Kondo" peak in $S(T)$ at $T=T_{p}$ is found to scale with $T_{K}$ [as defined in Eq. (19)], as shown in the inset of Fig. 3(d). Thus, in contrast to $T_{1}$ and $T_{2}, T_{p}$ can be considered a low-energy scale in the Kondo regime. The central positive peak in $S(T)$ first grows with positive magnitude on moving away from the Kondo regime [Fig. 3(d)] and then decreases in magnitude in Fig. 3(e) on entering the mixedvalence regime. Simultaneously, the Kondo peak in $S(T)$ acquires a large negative value while merging with the highenergy (negative) peak at $T \approx 6 \Gamma$ on entering the mixedvalence regime [Fig. 3(e)]. Well into the mixed-valence regime, the thermopower exhibits a single negative peak on a scale $\Gamma$ with a distinct shoulder at higher temperatures due to the peak at $T \approx 6 \Gamma$. This picture continues to hold in the empty-orbital regime [see Fig. 3(f)], with the shoulder at $T$ $\approx 6 \Gamma$ having almost disappeared. The thermopower remains negative for all gate voltages $v_{g}>0$ in this regime.

The above behavior in the temperature dependence of the thermopower in the Kondo regime is explained in terms of the structure of the single-particle excitations in $A(\omega, T)$. At low temperatures, a Sommerfeld expansion for $S(T)$ gives $^{15}$

$$
S(T)=-\left.\frac{k_{B}}{|e|} \frac{\pi^{2}}{3} k_{B} T \frac{1}{A(0, T)} \frac{\partial A}{\partial \omega}\right|_{\omega=0},
$$

showing that the sign of the thermopower depends on the slope of the spectral density at the Fermi level. For $T \ll T_{K}$ and $v_{g}>0$, the Kondo resonance lies above the Fermi level so the slope of the spectral density at the Fermi level is positive, resulting in a negative thermopower. This remains true on further increasing the temperature but as shown in Ref. 15, eventually the Kondo resonance is suppressed at $T$ $>T_{K}$ resulting in a negative slope of the spectral density at $\omega=0$ for $v_{g}>0$ (with the opposite being true for $v_{g}<0$ ). Consequently, the thermopower changes sign at the temperature $T_{1}$ which roughly corresponds to the temperature at which the Kondo resonance vanishes. At $T \gg T_{K}$, the determining factor for the sign of the thermopower is no longer the slope of the spectral function at $\omega=0$ but the number of states available below or above the Fermi level. These determine the overall sign of the transport integral $I_{1}$ in the expression for the thermopower in Eq. (5). For $v_{g}>0$, there are $n_{d} / 2<0.5$ states below the Fermi level and $1-n_{d} / 2>0.5$ states above the Fermi level. Consequently, the integral of $-(\partial f / \partial \omega) \omega A(\omega, T)$ for $\omega>0$ is greater than its counterpart for $\omega<0$ so $I_{1}>0$ and the thermopower is again negative at $T \gg T_{K}$. This occurs at $T=T_{2}$, which is found to be of order $\Gamma$ (see below). Due to the factor $1 / T$ coming from the derivative of the Fermi function in $I_{1}$, the negative thermopower at $T>T_{2}$ acquires a maximum negative value and then decreases as $1 / T$ at $T \gg \Gamma$, exhibiting no further sign changes, as confirmed also numerically. We note that, away from half filling $\left(v_{g}=0\right)$, the modified second-order perturbation in $U$ approach $^{50,51}$ gives an incorrect sign for the slope of the spectral density at the Fermi level in the Kondo regime. This results in a wrong sign for the thermopower at $T<T_{K}$ in the Kondo regime ${ }^{21}$ compared to our NRG calculations (which agree with those of Ref. 52). Approximate approaches using an infinite $U$ Anderson model ${ }^{53,54}$ could also not access the low-temperature Kondo regime.

The sign changes in the thermopower of strongly correlated quantum dots at the temperatures $T_{1}$ and $T_{2}$ in the Kondo regime, are particularly interesting. They provide a "smoking gun" signature for Kondo behavior in quantum dots, and could be used in future experiments as sensitive probes of strong correlations and Kondo physics. It is therefore interesting to give a detailed characterization of the dependence of $T_{1}$ and $T_{2}$ on gate voltage and interaction strength $U / \Gamma$. We show in Figs. 4(a) and 4(b) the loci of $T_{1}$ and $T_{2}$ as a function of $v_{g}>0$ for quantum dots and magnetic impurities for three interaction strengths. Although $S(T)$ vanishes at $v_{g}=0, T_{1}$ and $T_{2}$ have finite limiting values there. These are difficult to determine numerically due to the vanishingly small thermopower in this limit, and they are difficult to obtain analytically since $T_{1}$ and $T_{2}$ lie outside the Fermi-liquid regime where analytic calculations are possible. Estimates of these values at the smallest gate voltage are close to the limiting values. They are tabulated in Table I, together with the relevant Kondo scales at midvalley. Whereas the limiting values of $T_{1}$ are comparable for both quantum dots and magnetic impurities, the limiting values of $T_{2}$ for quantum dots are approximately twice larger than for magnetic impurities. By carrying out additional calculations, using a finer grid of gate voltages, we determined the critical gate voltages $v_{g}^{c}(U / \Gamma)$, beyond which no sign change occurs [indicated in Figs. 4(a) and 4(b)]. For each value of $U / \Gamma$, we find that $v_{g}^{c}$ corresponds to entering the mixed-valence re- 
TABLE I. Kondo temperature $T_{K}$ (in units of $\Gamma$ ) at midvalley $v_{g}=0$ (symmetric point) for $U / \Gamma=8,6,3$. Also shown are the temperatures $T_{1}$ and $T_{2}$ (in units of $\Gamma$ ) at which the thermopower, $S(T)$, changes sign at $v_{g}=0.25$ (the smallest $v_{g}$ studied). The numbers in brackets are the corresponding temperatures for magnetic impurities.

\begin{tabular}{cccc}
\hline \hline$U / \Gamma$ & $T_{K}\left(v_{g}=0\right) / \Gamma$ & $T_{1}\left(v_{g}=0.25\right) / \Gamma$ & $T_{2}\left(v_{g}=0.25\right) / \Gamma$ \\
\hline 8 & $2.64 \times 10^{-3}$ & $0.044(0.04)$ & $3.04(1.39)$ \\
6 & $1.10 \times 10^{-2}$ & $0.056(0.05)$ & $2.12(1.00)$ \\
3 & $8.20 \times 10^{-2}$ & $0.13(0.12)$ & $0.71(0.41)$ \\
\hline \hline
\end{tabular}

gime, i.e., $v_{g}^{c}$ corresponds to a local level position $\varepsilon_{d} \approx$ $-\Gamma / 2$ in the single-channel Anderson model.

\section{Thermal conductance: $K_{\mathrm{e}}(T)$}

The electronic contribution to the thermal conductance of a strongly correlated quantum dot, shown in Figs. 3(g)-3(i), also exhibits interesting behavior: a crossing point at $T \approx \Gamma$ is found in the Kondo regime and for gate voltages approaching the mixed-valence regime [Fig. 3(g) and inset]. Such (approximate) crossing points are typical signatures of strong correlations and are well known in other contexts, including ${ }^{3} \mathrm{He}$ and heavy fermions, ${ }^{55}$ dissipative two-level systems ${ }^{56}$ and doped Mott insulators. ${ }^{57}$ On entering the mixed-valence and empty-orbital regimes [Figs. 3(h) and 3(i)], two peaks develop on either side of the crossing point (the lower peak being at $T \lesssim 0.5 \Gamma$ and the upper one at $T \gtrsim 2 \Gamma)$. These qualitative features in $K_{\mathrm{e}}(T)$ can be related to $A(\omega, T)$, as in the case of $S(T)$ (see also Sec. VI C).

\section{Moderate to weak correlations}

The effect of reducing correlations to a moderate value, $U / \Gamma=3$, is shown in Fig. 11 of Appendix B: the trends are similar to those described above, with a significantly diminished Kondo regime. In particular, the evolution with gate voltage of $G(T)$ is similar to that in the strongly correlated case [see Figs. 11(a)-11(c)] and the thermopower exhibits two sign changes as a function of temperature in the Kondo regime [Fig. 11(d)], with a rapid evolution to a single negative peak in the mixed-valence and empty-orbital regimes [Figs. 11(e) and 11(f)]. However, the crossing point in $K_{\mathrm{e}}$ in the Kondo regime becomes less evident for moderate correlations [Fig. 11(g)], and, the two-peaked structure for $K_{\mathrm{e}}$ in the mixed-valence and empty-orbital regimes is replaced by a single peak with a shoulder [Figs. 11(h) and 11(i)].

These general trends, for correlated quantum dots, contrast with those for weakly correlated quantum dots, shown in Figs. 12(a)-12(c) of Appendix B for $U / \Gamma=1$. These exhibit no sign change in the thermopower for any gate voltage $v_{g}>0$. Similarly, the thermal conductance for weakly correlated quantum dots shows no crossing point, exhibiting only a single finite-temperature peak.

\section{E. High-temperature asymptotics}

The FDM approach allows us to easily investigate the high-temperature asymptotics of transport properties. As we

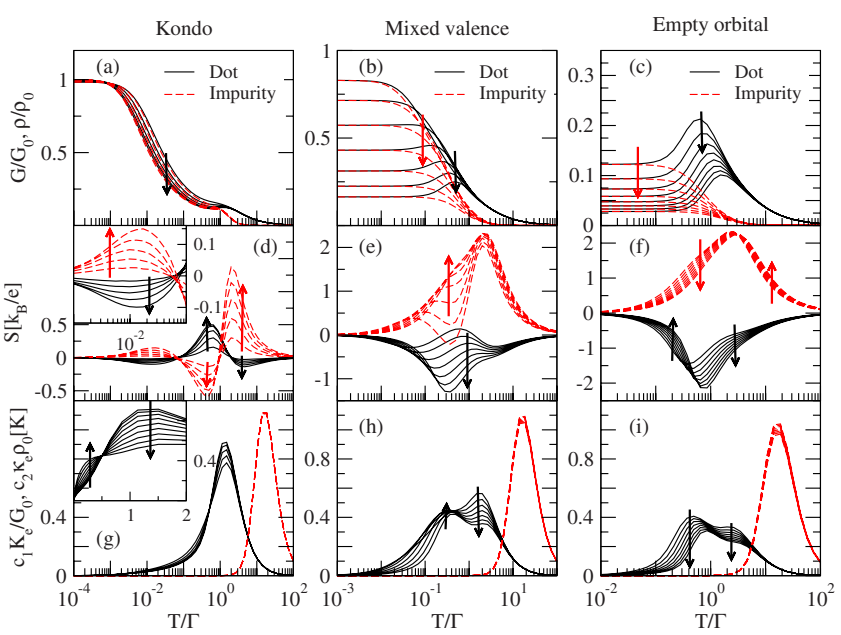

FIG. 5. (Color online) Comparison of transport properties for quantum dots (solid lines) and magnetic impurities (dashed lines) in the strongly correlated regime $U / \Gamma=6$. (a)-(c): normalized electrical conductance $G(T) / G_{0}$ (quantum dot) and normalized resistivities $\rho / \rho_{0}$ (impurity), where $\rho_{0}$ is defined in Eq. (16). (d)-(f): thermopower $S(T)$ for quantum dots and impurities [inset of (d) shows the low-temperature Kondo peak in the thermopower in more detail]. (g)-(i): electronic contribution to the thermal conductance $c_{1} K_{\mathrm{e}} / G_{0}$ (quantum dot) and thermal conductivity $c_{2} \kappa_{\mathrm{e}} \rho_{0}$ (impurity), rescaled by $G_{0}$ and $\rho_{0}$, respectively, so that the same unit $(K)$ applies to both cases. The numerical factors $c_{1}=10^{2}$ and $c_{2}=5$ $\times 10^{-4}$ are included for clarity of presentation. The inset in $(\mathrm{g})$ for $K_{\mathrm{e}}(T) / G_{0}$ shows the crossing point in $K_{\mathrm{e}}(T) / G_{0}$ at $T / \Gamma \approx 0.6$ in more detail and the evolution of the second peak in the thermal conductance of the quantum dot as the mixed-valence regime is approached (with inclusion of four additional gate voltages $v_{g}$ $=1.50, \ldots, 2.25$ in the Kondo regime). The range of gate voltages is $v_{g}=0.25, \ldots, 1.25$ (Kondo), $v_{g}=2.25, \ldots, 3.75$ (mixed valence), and $v_{g}=4.0, \ldots, 5.75$ (empty orbital).

discuss also in the context of dilute magnetic impurities in Sec. VI below, earlier transport calculations ${ }^{15}$ could not discern the highest-temperature peak in $S(T)$ [occurring at $T$ $\approx 6 \Gamma$ for $U / \Gamma=8$, see Fig. $3(\mathrm{~d})]$, nor the peak in the thermal conductivity (see discussion in Sec. VI below). Here, we are able to do so. In addition, the numerical calculations recover the high-temperature asymptotics of the transport properties: $G(T) \sim 1 / T, S(T) \sim 1 / T$, and $K_{\mathrm{e}}(T) \sim 1 / T^{2}$ for $T \gg \Gamma$. Note that, for the Anderson model, the logarithmic corrections in the Kondo regime occur at intermediate temperatures $T_{K}$ $\ll T \ll \Gamma$ : the corrections at $T \gg \Gamma$ go over to the above power laws.

\section{COMPARISON WITH DILUTE MAGNETIC IMPURITIES}

It is interesting to quantify the differences in the transport properties of quantum dots given by Eqs. (4)-(6) with the analogous transport properties of dilute magnetic impurities given by Eqs. (11)-(13). This is shown in Fig. 5 for the temperature dependence of transport properties in the Kondo, mixed-valence, and empty-orbital regimes for $U / \Gamma$ $=6$. 


\section{A. Comparison of $G(T)$ and $\rho(T)$}

In the Kondo regime, and for temperatures $T \ll \Gamma$, the conductance of a quantum dot is a universal function of $T / T_{K}$, i.e., $G(T) / G(0)=f\left(T / T_{K}\right)$ (e.g., see Ref. 47). The same holds for the analogous quantity for dilute magnetic impurities, namely, the resistivity, i.e., $\rho(T) / \rho(0)=f^{\prime}\left(T / T_{K}\right)$ (e.g., see Ref. 15). Since $G$ and $\rho$ are different physical quantities, the functions $f$ and $f^{\prime}$ are different and they cannot be made to coincide by using a common Kondo scale $T_{K}$ [e.g., the Kondo scale defined in Eq. (19)]. This is seen in Fig. 5(a), which shows that the conductance curves for quantum dots are shifted in temperature, on a logarithmic scale, relative to the resistivity curves of magnetic impurities. The two functions $f$ and $f^{\prime}$ are rigorously identical only in the Fermiliquid regime $T \ll T_{K}$. Experimentally, however, the accessible range of temperatures is that around $T \approx T_{K}$, say one decade below and one decade above $T_{K}$. For this region of temperatures, the two functions $f$ and $f^{\prime}$ can be made to coincide by redefining them as new functions $\tilde{f}$ and $\tilde{f}^{\prime}$, respectively, with different respective Kondo scales, $T_{K}^{\mathrm{G}}$ and $T_{K}^{\rho}$ such that $\tilde{f}\left(T / T_{K}^{\mathrm{G}}=1\right)=\tilde{f}^{\prime}\left(T / T_{K}^{\rho}=1\right)=1 / 2$, see Ref. 47 . In the mixed-valence and empty-orbital regimes, Figs. 5(b) and 5(c) show that the conductance of a quantum dot differs significantly from the resistivity of magnetic impurities (with significant deviations at $T \gtrsim 0.1 \Gamma$ ). In particular, the aforementioned finite-temperature peak in the conductance of a quantum dot is absent in the resistivity of magnetic impurities. A signature of this peak in $\rho(T)$ is seen at most in the Kondo regime at temperatures of order $\Gamma$ [see Fig. 5(a)] and is absent in the mixed-valence and empty-orbital regimes. These differences to the quantum dot case, arise, as described in Sec. III, due to the different way in which the spectral function appears in the respective transport integrals. These differences reflect also the absence of universality outside the Kondo regime.

\section{B. Comparison of thermopowers: $S(T)$}

In Figs. 5(d)-5(f) we see that, up to an overall sign change, due to $A(\omega, T)$ appearing differently in the transport integrals as explained in Sec. III, the thermopower of magnetic impurities behaves in a qualitatively similar way to that of a quantum dot, with two sign changes at $T_{1}\left(v_{g}\right)$ and $T_{2}\left(v_{g}\right)$ [shown in Fig. 4(b)] and three extrema. In the Kondo regime, the position, $T_{p}$, of the Kondo enhanced peak in the thermopower of magnetic impurities is found to scale with $T_{K}$, just as for the quantum dot case (see Sec. V B). A significant difference between $S(T)$ for magnetic impurities and quantum dots is the much larger high-temperature peak (at $T$ $>T_{2}$ ) for the former in the Kondo regime [by as much as a factor 5, see Fig. 5(d)]. This difference holds to some extent also in the mixed-valence regime [Fig. 5(e)]. In the emptyorbital regimes, the thermopowers show a single peak at $T$ $\approx \Gamma$ with a similar magnitude for both cases [Fig. 5(f)].

\section{Comparison of $K_{\mathrm{e}}(T)$ and $\kappa_{\mathrm{e}}(T)$}

The electronic contribution to the thermal conductivity of magnetic impurities, $\kappa_{\mathrm{e}}$, shows significant differences to the corresponding thermal conductance of quantum dots, $K_{\mathrm{e}}$, see Figs. 5(g)-5(i). For example, whereas $K_{\mathrm{e}}$ exhibits interesting structure with either one (in the Kondo regime) or two (in the mixed-valence and empty-orbital regimes) peaks around $T$ $\approx \Gamma, \kappa_{\mathrm{e}}$ only exhibits a single peak in all regimes, and this peak occurs at a much larger temperature $T \gg \Gamma$. The reason for the latter difference is the following: the main contribution to the thermal conductance and thermal conductivity come from the integrals $I_{2}$ and $M_{2}$ in Eqs. (7) and (14), respectively, which involve integrals of $-(\partial f / \partial \omega) \omega^{2} A(\omega, T)$ and $-(\partial f / \partial \omega) \omega^{2} / A(\omega, T)$, respectively. For the former, the peaks in $A(\omega, T)$ at $\varepsilon_{d}$ and $\varepsilon_{d}+U$ result in a peak in the integrand at $|\omega| \geqslant U / 2 \geqslant \Gamma$ whereas in the latter, the dips in $1 / A(\omega, T)$ at $\varepsilon_{d}$ and $\varepsilon_{d}+U$ shift the main contribution to the integral to much higher energies $|\omega| \gg U / 2$. Correspondingly, the temperature of the peaks in $K_{\mathrm{e}}$ in the former are at $T$ $\lesssim U / 2$ and for the latter are at $T \gg U / 2>\Gamma$, in agreement with the numerical results. The existence of two peaks in $K_{e}$ in the mixed-valence and empty-orbital regimes as opposed to a single peak in the Kondo regime is also easily explained: the two peaks reflect the sampling of the two incoherent features at $\varepsilon_{d}$ and $\varepsilon_{d}+U$ in $\omega^{2} A(\omega, T)$ appearing in the moment $I_{2}$ for $K_{\mathrm{e}}$. In the Kondo regime, these excitations lie close to each other and only one peak results. Similarly, the single peak in $\kappa_{\mathrm{e}}$ for all regimes results from the strong suppression of the above incoherent excitations in $1 / A(\omega, T)$ appearing in the moment $M_{2}$.

\section{FIGURE OF MERIT, POWER FACTOR, AND LORENZ NUMBER}

A measure of the thermoelectric efficiency of a quantumdot device is the dimensionless figure of merit defined by $Z T=G S^{2} T /\left(K_{\mathrm{e}}+K_{\mathrm{ph}}\right)$, where $K_{\mathrm{ph}}$ is the phonon contribution to the thermal conductance. Hence for high efficiency, one requires either large $S$ or small total thermal conductance relative to electrical conductance or both conditions simultaneously. A calculation of ZT for quantum-dot systems would therefore require knowledge of the material-specific phonon contribution to the thermal conductance $K_{\mathrm{ph}}$. Similarly, for magnetic impurity systems, a calculation of the dimensionless figure of merit, $Z T=\sigma S^{2} T /\left(\kappa_{\mathrm{e}}+\kappa_{\mathrm{ph}}\right)$, would require knowledge of the material-specific phonon contribution to the thermal conductivity $\kappa_{\mathrm{ph}}$. This is outside the scope of the present paper, so instead we show in Figs. 6(a)-6(c) results at $U / \Gamma=8$ for the quantity $Z T_{0}=G S^{2} T / K_{\mathrm{e}}$, for quantum dots, and $Z T_{0}=\sigma S^{2} T / \kappa_{\mathrm{e}}$, for magnetic impurities (with the latter being depicted on the negative axis for clarity). In addition, we also show in Figs. 6(d)-6(f) an appropriate rescaled power factor $\left(P F_{0}=S^{2} G / G_{0}\right.$ for quantum dots and $P F_{0}$ $=S^{2} \sigma / \sigma_{0}$ for magnetic impurity systems). This is another useful measure of an efficient thermoelectric system, bypassing lack of knowledge of the total thermal conductances (conductivities).

\section{A. Figure of merit}

Since, in the low-temperature limit, the thermopower of the Anderson model vanishes linearly with temperature in all 

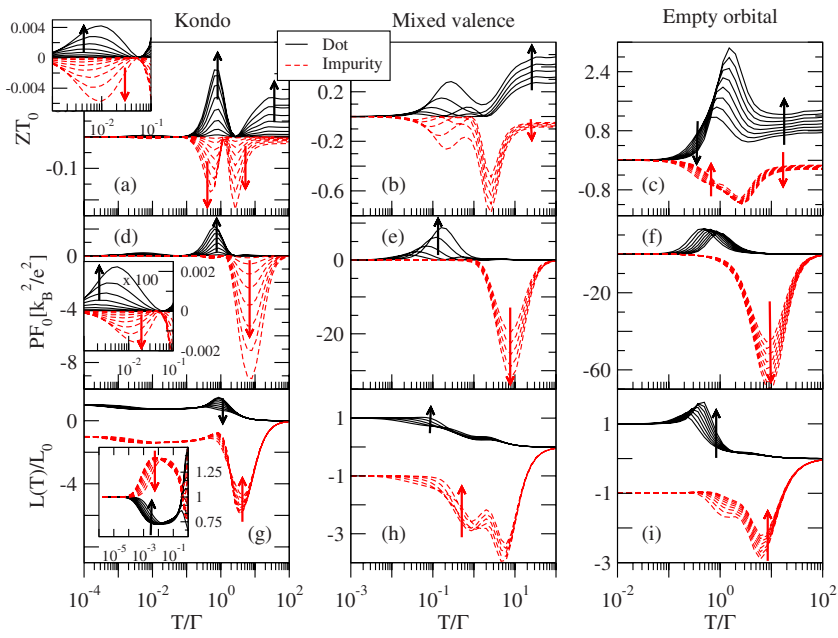

FIG. 6. (Color online) Temperature dependence of, (a)-(c), the "figure of merit," $Z T_{0},(\mathrm{~d})-(\mathrm{f})$, the "power factor," $P F_{0}$, and, (g)-(i), the Lorenz number ratio $L(T) / L_{0}$ for a quantum dot (solid lines, positive $y$ axis) and for magnetic impurities (dashed lines, shown on the negative $y$ axis for clarity). Results are for $U / \Gamma=8$ and the same range of dimensionless gate voltages as in Fig. 3. For quantum dots we define $Z T_{0}=G S^{2} T / K_{\mathrm{e}}, P F_{0}=S^{2} G / G_{0}$, and $L(T) / L_{0}=K_{\mathrm{e}} / G T$ with $L_{0}=\pi^{2} k_{B}^{2} / 3 e^{2}$. The corresponding quantities for magnetic impurities are defined by $Z T_{0}=\sigma S^{2} T / \kappa_{\mathrm{e}}, P F_{0}=S^{2} \sigma / \sigma_{0}$, and $L(T) / L_{0}$ $=\left(\kappa_{\mathrm{e}} / \sigma T\right)$ where $\sigma=1 / \rho$ and $\sigma_{0}=1 / \rho_{0}$. Arrows indicate the evolution of the transport quantities with increasing $v_{g}>0$. Insets in (a) and (d) for the Kondo regime, show the low-temperature peak in $Z T_{0}$ and $P F_{0}$ in the vicinity of $T_{K}\left(v_{g}\right) \geqslant 2.6 \times 10^{-3} \Gamma$ (Table I). The inset in $(\mathrm{g})$ for the Lorenz number shows the deviations from the Wiedemann-Franz law in the region around $T_{K}\left(v_{g}\right)$. In this inset, $L(T) / L_{0}$ is shown on the positive $y$ axis for both impurity and quantum-dot cases.

regimes, a significant figure of merit is found only at finite temperature, as seen in Figs. 6(a)-6(c).

In the Kondo regime, Fig. 6(a), enhanced regions of $Z T_{0}$ are found in four temperature regions, (i), at $T \approx T_{p}$, due the low-temperature Kondo enhancement of the thermopower, however the magnitude of $Z T_{0}$ is tiny [see inset of Fig. 6(a)], (ii), at temperatures of order $\Gamma$ in the region $T_{1}<T<T_{2}$, where $Z T_{0}$ can be of order $0.2-0.3$ for both quantum dots and magnetic impurities, (iii), at temperatures $T>T_{2}$, with enhancements comparable to those for region (ii), and, (iv), in the asymptotic region $T \gg \Gamma$, where $Z T_{0}$ saturates to a finite value which is larger for quantum dots than for magnetic impurities (discussed below).

The behavior of the figure of merit in the mixed-valence and empty-orbital regimes is complicated, see Figs. 6(b) and 6(c). In the mixed-valence regime, significant enhancements are found, for quantum dots, at temperatures somewhat below $\Gamma$, see Fig. 6(b), and in the asymptotic regime $T \gg \Gamma$. Similar enhancements are found also for the empty-orbital case [Fig. 6(c)]. For magnetic impurities, similar enhancements to quantum dots are found on temperature scale of order $\Gamma$ but at $T \gg \Gamma$, the enhancements are much smaller than for quantum dots [see Figs. 6(b) and 6(c)]. The latter effect is due to the much larger thermal conductivities (even at higher temperatures) of magnetic impurities as compared to those of quantum dots [see discussion above and Figs. $6(\mathrm{~g})-6(\mathrm{i})]$.

\section{B. Power factor}

The power factor $P F_{0}$ is enhanced in the same regimes (i)-(iii) as the figure of merit, see Figs. 6(e) and 6(f), but vanishes as $1 / T^{3}$ in the limit $T \gg \Gamma$ (using the asymptotic behavior of $S$ and $G$ from Sec. V E). In the Kondo regime, $P F_{0}$ exhibits a much larger peak above $T_{2}$ for magnetic impurities as compared to quantum dots [see Fig. 6(d)]. This reflects the observation made above (Sec VIB) that the highest-temperature peak in $S(T)$ at $T>T_{2}$ for magnetic impurities is significantly enhanced as compared to that of quantum dots. For quantum dots, the main enhancement in $P F_{0}$ in the Kondo regime is in the range $T<T_{2}$. These trends differ little from those observed in the mixed-valence and empty-orbital regimes for both quantum dots and magnetic impurities [see Figs. 6(e) and 6(f)].

\section{Wiedemann-Franz law and Lorenz number}

We comment on the enhancement of $Z T_{0}$ in the region $T \gg \Gamma$, which can result in $Z T_{0}>1$ (e.g., in the mixed-valence and empty-orbital cases). This enhancement reflects a violation of the Wiedemann-Franz law at $T \gg \Gamma$. The latter states that the thermal conductance (conductivity) is proportional to the electrical conductance (conductivity) multiplied by temperature, i.e., that the Lorenz number $L(T)$, defined for quantum dots by

$$
L(T)=K_{\mathrm{e}}(T) / T G(T),
$$

and for magnetic impurities by

$$
L(T)=\kappa_{\mathrm{e}}(T) / T \sigma(T),
$$

is independent of temperature and takes on the universal value $L_{0}=\pi^{2} k_{B}^{2} / 3 e^{2}$. Since, $Z T_{0}=S^{2} / L(T)$, a significant reduction in $L(T) / L_{0}$ can result in an enhancement of $Z T_{0}$. In Figs. 6(g)-6(i), we see that $L(T) / L_{0}$ is much suppressed at $T \gg \Gamma$, thereby allowing for significant enhancements in $Z T_{0}$ in this limit. This enhancement is seen for all regimes, especially for the mixed-valence and empty-orbital regimes. We note, however, that from Figs. 6(g)-6(i) (and inset), the Wiedemann-Franz law is, on the whole, reasonably well satisfied at temperatures $T \ll \Gamma$, and becomes exact in the Fermiliquid regime ${ }^{15}$ (for other violations of the Wiedemann-Franz law see Ref. 58).

\section{UNIVERSAL SCALING FUNCTIONS FOR THERMAL TRANSPORT THROUGH QUANTUM DOTS}

By analogy to the scaling properties of the electrical conductance $G(T) / G(0)=f\left(T / T_{K}\right)$, where $f$ is a universal function of $t=T / T_{K}$ in the Kondo regime, it is interesting to establish to what extent such scaling is present in the thermopower, $S(T)$, and the thermal conductance, $K_{\mathrm{e}}(T)$, of strongly correlated quantum dots. We investigate this here, for $U / \Gamma=8$ and for values of the gate voltage in the Kondo regime (see Fig. 7). 


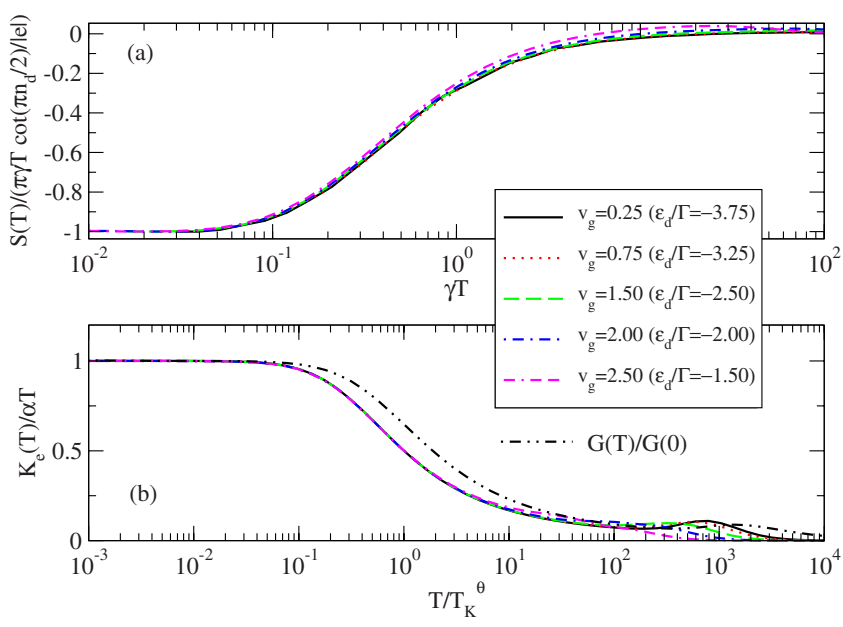

FIG. 7. (Color online) (a) $S(T) / T$ for a quantum dot, scaled by its limiting low-temperature absolute value $\pi \gamma \cot \left(\pi n_{d} / 2\right) /|e|$ versus $\gamma T \sim T / T_{K}$ for $U / \Gamma=8$ and a range of gate voltages in the Kondo regime. Here, $\gamma \sim 1 / T_{K}$ (Ref. 59). (b) The thermal conductance $K_{\mathrm{e}}(T)$, scaled by $\alpha T$, versus $T / T_{K}^{\theta}$, for parameters as in (a), with $\alpha$ defined in Eq. (24) and $T_{K}^{\theta}$ a Kondo scale defined in Eq. (25). The electrical conductance $G(T) / G(0)$ versus $T / T_{K}^{\theta}$ at $v_{g}=0.25$ is also shown. The Kondo scale defined by $G\left(T=T_{K}\right)=G(0) / 2$ is related to $T_{K}^{\theta}$ by $T_{K} \approx 1.9 T_{K}^{\theta}$.

In the Fermi-liquid regime, $T \ll T_{K}$, we have ${ }^{15}$

$$
S(T)=-\frac{\pi \gamma T}{|e|} \cot \left(\pi n_{d} / 2\right) .
$$

Scaling can therefore be expected for $S(T) / T$, once the occupancy (and gate voltage) dependent factor $\cot \left(\pi n_{d} / 2\right)$ is scaled out. In the above, $\gamma \sim 1 / T_{K}$ is a measure of the inverse Kondo scale and can be extracted ${ }^{59}$ from the numerical value of $\lim _{T \rightarrow 0}|S(T)| / T$ using Eq. (23) and the calculated values of $n_{d}$ from Fig. 1. We see from Fig. 7(a) that $s\left(T / T_{K}\right)$ $=|e| S(T) / \pi \gamma T \cot \left(\pi n_{d} / 2\right)$ does indeed scale with $\gamma T$ $\sim T / T_{K}$ for a range of gate voltages in the Kondo regime. This scaling extends up to temperatures comparable to $T_{K}$ with significant deviations setting in above this temperature scale. This is not surprising given the fact that the thermopower is a highly sensitive probe of the particle-hole asymmetry in the spectral density.

For the thermal conductance, $K_{\mathrm{e}}(T)$, we expect from the Wiedemann-Franz law, $K_{\mathrm{e}} / T \sim G(T)$, to see a scaling in $K_{\mathrm{e}}(T) / T$ similar to that in $G(T) / G(0)$. This is confirmed in Fig. 7(b) which shows $K_{\mathrm{e}}(T) / \alpha T$ versus $T / T_{K}^{\theta}$ for several gate voltages in the Kondo regime, where $\alpha$ is defined by

$$
\alpha=\lim _{T \rightarrow 0} \frac{K_{\mathrm{e}}(T)}{T}=\frac{\pi^{2} k_{B}^{2} A(0,0)}{3}
$$

and $T_{K}^{\theta}$ is a Kondo scale defined by

$$
\frac{K_{\mathrm{e}}\left(T=T_{K}^{\theta}\right)}{T_{K}^{\theta}}=\frac{\alpha}{2} .
$$

We see that, for $U / \Gamma=8, K_{\mathrm{e}}(T) / \alpha T=g\left(T / T_{K}^{\theta}\right)$ is a universal function of $T / T_{K}^{\theta}$ for temperatures extending up to at least $100 T_{K}^{\theta}$, just as $G(T) / G(0)=f\left(T / T_{K}\right)$ is a universal function of
$T / T_{K}$ for temperatures extending up to at least $100 T_{K}$. Increasing $U / \Gamma$, and thereby reducing $T_{K} / \Gamma$ allows universality to extend to still higher temperatures. Suppressing charge fluctuations, e.g., by working within a Kondo model, allows these universal scaling functions to be defined for all temperatures. Note also, that although these universal functions $f$ and $g$ have a similar functional dependence on $T / T_{K}$ and $T / T_{K}^{\theta}$, respectively, they are shifted relative to one another on an absolute-temperature scale. The difference between $g$ and $f$ for temperatures around $T=T_{K}^{\theta} \approx T_{K} / 1.9$ accounts for the violation in the Wiedemann-Franz law on this scale, as noted previously [see inset of Fig. 6(g)]. The Wiedemann-Franz law is only satisfied exactly in the Fermi-liquid regime $T$ $\ll T_{K}$. One can collapse $G(T)$ onto $K_{\mathrm{e}}(T) / T$ by scaling the temperature axis of the former by $T_{K}^{\theta} / T_{K}$. In the universal regime $T \ll \Gamma$, small deviations between $g(t)$ and $f(t)$ arise for $t \lesssim 1$ and $t \gtrsim 1$.

For dilute magnetic impurities, our conclusions for scaling in the Kondo regime are essentially the same as those above for quantum dots (see also Ref. 15).

\section{GATE-VOLTAGE DEPENDENCE OF TRANSPORT PROPERTIES}

The gate-voltage dependence of transport through quantum dots and magnetic impurities is shown for several representative temperatures in Fig. 8 for the electrical and thermal transport and in Fig. 9 for the thermopower. For magnetic impurities, different $v_{g}$ should be understood as corresponding to changes in the local level position $\varepsilon_{d}$ relative to the Fermi level, as invoked by the application of pressure (either chemical via doping or hydrostatic). In some rare-earth systems, ${ }^{18}$ the application of pressure has been shown to tune the magnetic impurities from the Kondo to the mixed-valence and empty-orbital regimes. Throughout this section, we denote by $T_{1}$ and $T_{2}$ the minimum temperatures of $T_{1}\left(v_{g}\right)$ and $T_{2}\left(v_{g}\right)$ in the limit $v_{g} \rightarrow 0$ (see Fig. 4 and Table I). We show that the different behavior in the gate-voltage dependence of the thermopower, at fixed temperature $T$, can be classified in terms of the relative value of $T$ to $T_{1}$ and $T_{2}$.

\section{A. Gate-voltage dependence of $G$ and $K_{\mathrm{e}}$}

The gate-voltage dependence of the electrical and thermal conductance of quantum dots is shown in Figs. 8(a)-8(c). The former exhibits, for $T \gg T_{K}$, Coulomb blockade peaks at $\varepsilon_{d}= \pm U / 2 \Gamma$ with a suppression of the conductance in the midvalley region around $v_{g}=0$. On decreasing the temperature, the Kondo effect becomes operative resulting in an enhancement of the conductance in the region between the Coulomb-blockade peaks [see Fig. 8(a)]. This picture is well known. At $T \gg T_{K}$, the thermal conductance of quantum dots also exhibits Coulomb-blockade peaks, ${ }^{60,61}$ not directly evident in the plot of $K_{\mathrm{e}}$ versus gate voltage [Fig. 8(b)], where only weak signatures of these are discernible. They become clearer in the gate-voltage dependence of $K_{\mathrm{e}} / T$ which by the Wiedemann-Franz law (see Sec. VII C) is proportional to the electrical conductance $G(T)$, as seen in Fig. 8(c). Differences between Figs. 8(a) and 8(c) indicate the degree of deviation 

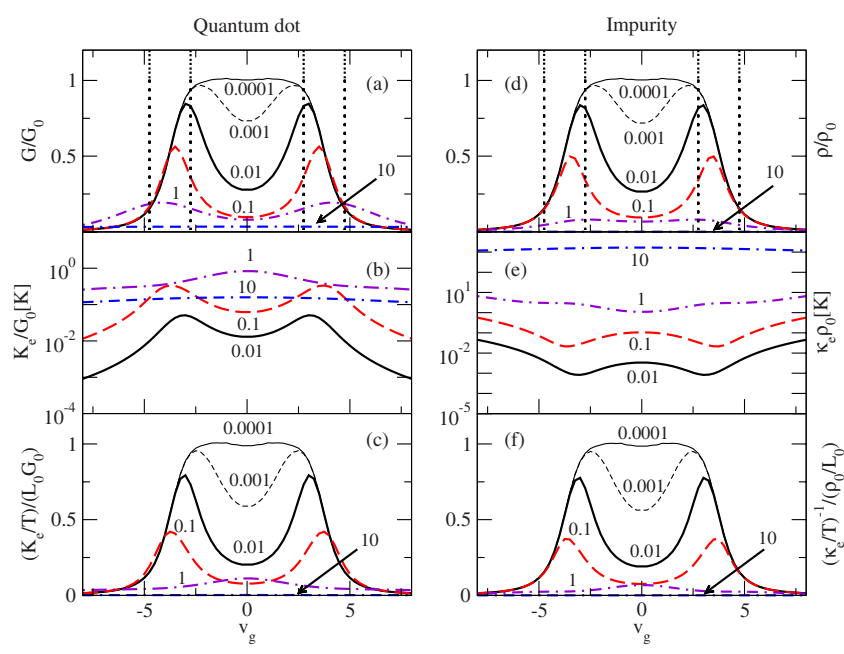

FIG. 8. (Color online) Gate-voltage dependence of electrical and thermal transport properties of quantum dots [left panels (a)-(c)] and magnetic impurities [right panels (d)-(f)] for $U / \Gamma=8$ and at several representative temperatures $T / \Gamma$ (labeling the individual curves in the figures). Vertical dashed lines in the top panels delineate the Kondo regime from mixed-valence and empty-orbital regimes. (a): The normalized conductance $G(T) / G_{0}$, (d): the normalized resistivity $\rho(T) / \rho_{0}$ for the impurity case, and, (b), the rescaled thermal conductance $K_{\mathrm{e}}(T) / G_{0}$, [(e): the rescaled thermal conductivity $\kappa_{\mathrm{e}}(T) \rho_{0}$ for the impurity case]. The bottom panel, (c), shows the dimensionless ratio $\left(K_{\mathrm{e}} / T\right) /\left(L_{0} G_{0}\right)$ for the quantum dot [panel (f) shows the analogous dimensionless ratio for magnetic impurities $\left.\left(\kappa_{\mathrm{e}} / T\right)^{-1} /\left(\rho_{0} / L_{0}\right)\right]$. Deviations from $G(T) / G_{0}$ in (a) reflects deviations from the Wiedemann-Franz law [similarly, deviations of $\left(\kappa_{\mathrm{e}} / T\right)^{-1} /\left(\rho_{0} / L_{0}\right)$ in (f) from $\rho(T) / \rho_{0}$ in (d) indicate deviations from the Wiedemann-Franz law for the impurity case]. For clarity not all temperatures are shown in (b) and (e).

from the Wiedemann-Franz law. These deviations are largest at $T \gg \Gamma$ for all gate voltages, as previously observed in Figs. $6(\mathrm{~g})-6(\mathrm{i})$.

The same observations, using a different terminology, can be made for the case of magnetic impurities in Figs.

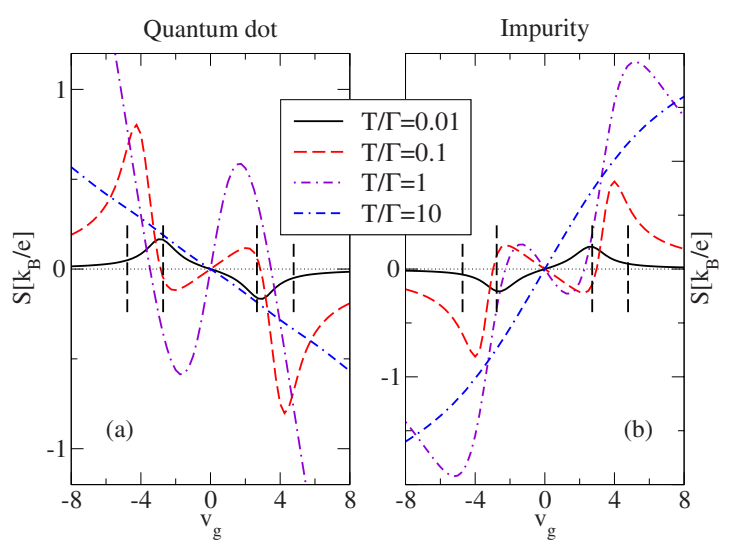

FIG. 9. (Color online) Gate-voltage dependence of the thermopower for the strong correlation case $U / \Gamma=8$ at several characteristic temperatures $T / \Gamma$ for, (a), the quantum dot case, and, (b), the impurity case. The vertical dashed lines delineate the Kondo regime (around $v_{g}=0$ ) from the surrounding mixed-valence and empty-orbital regimes. 8(d)-8(f): the valence fluctuation peaks at $T \gg \Gamma$ are now seen in the resistivity of mixed-valence impurities whereas Kondo impurities $\left(v_{g} \approx 0\right)$ have a small resistivity at $T \gg \Gamma$ and a large unitary resistivity at $T=0$. The behavior of the thermal conductivity $\kappa_{\mathrm{e}}$ is similarly understood in terms of the Wiedemann-Franz law $\kappa_{\mathrm{e}}(T) \sim \sigma T$ with $\sigma=1 / \rho$, as seen by comparing Figs. 8(d) and 8(f).

\section{B. Gate-voltage dependence of $S$}

The gate-voltage dependence of the thermopower of quantum dots is shown in Fig. 9(a). The particle-hole symmetry about $v_{g}=0$ (see Sec. II) implies $S_{-v_{g}}=-S_{v_{g}}$ at all temperatures so we only discuss $v_{g}>0$. We focus mainly on the Kondo regime, $v_{g}<v_{g}^{c}$, and discuss the remaining gate voltages by reference to Fig. 3. There are three main types of behavior, characterized by the following temperatures, $T$, relative to $T_{1}$ and $T_{2}$ : (i), $T<T_{1}$, as exemplified by $T$ $=0.01 \Gamma$, (ii), $T_{1}<T<T_{2}$ as exemplified by $T=0.1 \Gamma$ and $T$ $=\Gamma$, and, (iii), $T>T_{2}$, as exemplified by $T=10 \Gamma$. In case (i), the Kondo resonance is asymmetric about the Fermi level, ${ }^{15}$ lying slightly above it for $v_{g}>0$. The slope of the spectral density at $\omega=0$ is positive, resulting by Eq. (20) in a negative thermopower, as observed for $T=0.01 \Gamma$. The same holds, at still lower temperatures, $T \ll T_{K}$, where Fermi-liquid theory ${ }^{15}$ gives the explicit expression (23). Case (ii), $T_{1}<T<T_{2}$, is the most interesting for quantum dots, for several reasons: first, this temperature range is experimentally accessible since for $U / \Gamma=8$, we have $T_{1}=0.044 \Gamma$ and $T_{2}=3.04 \Gamma$. Second, there is an overall sign change in $S\left(v_{g}\right)$, relative to case (i), for a finite range of gate voltages [see Fig. 9(a)]. Third, a further sign change occurs at finite $v_{g}>0$, and, fourth, the thermopower is large enough for a significant range of gate voltages to enable its measurement. The sign change at a finite gate voltage occurs when $S\left(T_{1}<T<T_{2}\right)$ as a function of $v_{g}$ in Fig. 3(d) reaches the value zero and becomes negative.

For gate voltages outside the Kondo regime, the thermopower, as a function of gate voltage, $S\left(v_{g}\right)$, either approaches zero at $v_{g} \gg 1$ [as happens for $T=0.1$ in Fig. 9(a)] or does not saturate for $v_{g} \gg 1$ [e.g., for $T=\Gamma$ in Fig. 9(a)]. In terms of Fig. 3(f) (see the arrows), the former occurs for temperatures to the left of the minimum in $S$ in Fig. 3(f), and the latter occurs for the opposite case. The latter case is half way to case (iii), $T>T_{2} \gg \Gamma$, which exhibits a thermopower approximately linear in gate voltage, with no sign change at any $v_{g}>0$. This is similar to the "sawtooth" behavior of $S\left(v_{g}\right)$ found for multilevel quantum dots weakly coupled to leads at $T \gg \Gamma$ in Refs. 62 and 63 (for related experimental work see Refs. 64-69). The behavior of the thermopower in multilevel open quantum dots has also been investigated. ${ }^{70-72}$

The same classification (i)-(iii), as for quantum dots, can be used to explain the local level dependence of the thermopower of magnetic impurities shown in Fig. 9(b).

\section{CONCLUSIONS AND DISCUSSION}

In this paper, we investigated the thermoelectric properties of strongly correlated quantum dots, described by the 
single-level Anderson impurity model connected to two conduction-electron leads. For this purpose, we used Wilson's NRG method and calculated the local Green's function and transport properties by using the full density-matrix approach. ${ }^{33}$ Since this approach builds into the density matrix all excitations obtained in the NRG approach, it is particularly well suited to finite-temperature transport calculations, allowing us, for example, to investigate also the hightemperature asymptotics of transport properties.

For strong correlations and in the Kondo regime, the thermopower exhibits two sign changes, at temperatures $T_{1}\left(v_{g}\right)$ and $T_{2}\left(v_{g}\right)$ with $T_{1}<T_{2}$. We found that $T_{1}>T_{p}\left(v_{g}\right) \approx T_{K}\left(v_{g}\right)$, where $T_{p}\left(v_{g}\right)$ is the position of the Kondo-induced peak in the thermopower, $T_{K}\left(v_{g}\right)$ is the Kondo scale, and $T_{2}=O(\Gamma)$. The loci of $T_{1}\left(v_{g}\right)$ and $T_{2}\left(v_{g}\right)$ merge at a critical gate voltage $v_{g}=v_{g}^{c}(U / \Gamma)$, beyond which no sign change occurs. We determined $v_{g}^{c}$ for different $U / \Gamma$ finding that $v_{g}^{c}$ coincides, in each case, with entry into the mixed-valence regime. No sign change is found outside the Kondo regime or for weak correlations, $U / \Gamma \lesssim 1$. Thus, a sign change in $S(T)$ at finite $v_{g}$ is a particularly sensitive signature of strong correlations and Kondo physics. This effect could be measurable in quantum dots, as it manifests itself in an overall sign change in $S$ for a finite range of gate voltages on increasing temperature $T$ from below $T_{1}\left(v_{g} \rightarrow 0\right)$ to values in the range $T_{1}<T<T_{2}$ $=O(\Gamma)$, which is an accessible range since $T_{1}>T_{K}$.

The results for quantum dots were compared also to the relevant transport coefficients of dilute magnetic impurities in nonmagnetic metals: the electronic contribution, $\kappa_{\mathrm{e}}$, to the thermal conductivity, the thermopower, $S$, and the impurity contribution to the electrical resistivity, $\rho$. As regards the temperature dependence of the respective transport quantities, we find, in the mixed-valence and empty-orbital regimes, two peaks in $K_{\mathrm{e}}(T)$ as compared to a single peak in $\kappa_{\mathrm{e}}(T)$. Similarly, $G(T)$ exhibits a finite-temperature peak on entering the mixed-valence regime whereas such a pronounced peak is absent in $\rho(T)$, even far into the emptyorbital regime. As for quantum dots, we find that the lowtemperature Kondo peak position in the thermopower of magnetic impurities scales with $T_{K}$. We compared and contrasted the figure of merit, power factor, and the extent of violation of the Wiedemann-Franz law in quantum dots and dilute magnetic impurities, finding enhanced figures of merit at temperatures where the Wiedemann-Franz law is strongly violated. Finally, we clarified the extent of scaling, as a function of $T / T_{K}$, in the thermopower and thermal conductance of quantum dots in the Kondo regime.

We comment on a recent experiment in Ref. 20 which we believe shows evidence of Kondo correlations in the thermopower of a strongly correlated quantum dot. In this experiment, the thermovoltage across a Kondo correlated quantum dot is investigated as a function of gate voltage and lattice temperature. This can be compared to our $S\left(v_{g}\right)$ in Fig. 9(a). The gate voltage $V_{E}$ in Ref. 20 is related to our dimensionless gate voltage, $\mathrm{v}_{g}$, via $-e V_{E} \sim \varepsilon_{d} \sim v_{g}$, i.e., $V_{E} \sim-v_{g}$. Mirror reflecting our results for $S\left(v_{g}\right)$ in Fig. 9(a) about $v_{g}$ $=0$ allows a qualitative comparison with the experimental measurements in Ref. 20. Using the experimental estimate $\Gamma=0.35 \mathrm{meV}$ from Ref. 20, we can translate the four experimental temperatures $T_{L}=0.07 \mathrm{~K}, 0.25 \mathrm{~K}, 1.00 \mathrm{~K}$ and $T_{L}$

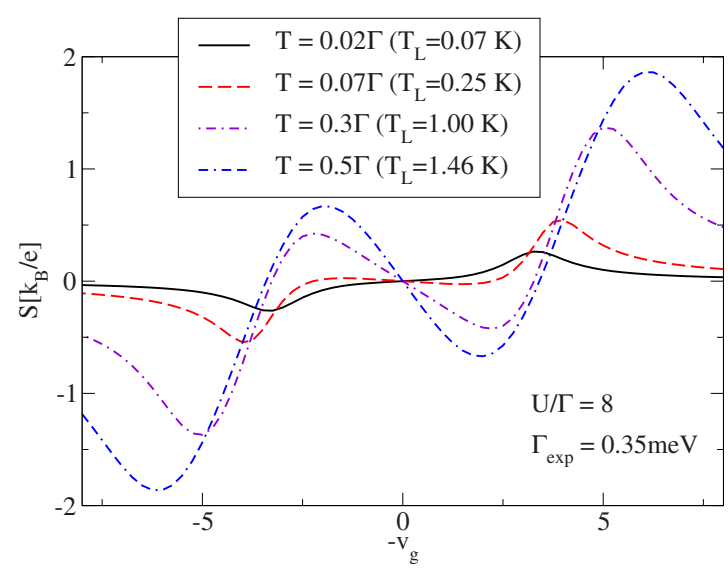

FIG. 10. (Color online) Gate-voltage dependence of the thermopower for $U / \Gamma=8$ at temperatures $T / \Gamma$ corresponding to the experimental ones $T_{L}$ from Ref. 20 using $\Gamma_{\exp }=0.35 \mathrm{meV}$. Since the experimental gate voltage $V_{E} \sim-v_{g}$ we plotted $S$ versus $-v_{g}$ to facilitate comparing with the experimental data.

$=1.46 \mathrm{~K}$ at which the thermopower was measured into our theoretical temperatures in units of $\Gamma$. We assume strong Coulomb correlations on the $\operatorname{dot} U / \Gamma=8$ and show the results for the thermopower in Fig. 10. From Table I, the lowest experimental temperature corresponds to $T \ll T_{1}$, the next lowest temperature $(T=1.00 \mathrm{~K})$ lies close to $T=T_{1}$, where the thermopower changes sign in the Kondo regime, and the highest two temperatures lie between $T_{1}$ and $T_{2}$. The lowest temperature measured, $T=0.07 \mathrm{~K}$, indeed shows a positive thermopower above midvalley, in agreement with our results for $T<T_{1}$. Upon increasing the temperature, the experiment shows a sign change in the thermovoltage for a finite range of gate voltages (relative to midvalley), which is consistent with our prediction of such a sign change in the Kondo regime for $T_{2}>T>T_{1}$. The onset, with increasing temperature, of an additional oscillation in $S\left(v_{g}\right)$ about $v_{g}=0$ in the experiments is therefore consistent with our results. The experimental data deviates from our calculated thermopower in the mixed-valence and empty-orbital range of gate voltages $v_{g}$ $\gg 1$, with the theoretical results showing a much larger thermopower in this region of gate voltages. These deviations are expected at $v_{g} \gg 1$ since additional levels present in real quantum dots, but absent in our model, start being populated. This significantly influences transport through the quantum dot. Qualitatively, however, we are able to interpret these experiments on the thermopower of Kondo correlated quantum dots for gate voltages $v_{g} \approx 0$. For a more quantitative comparison to theory, further investigations are needed.

Calculations for single quantum dots and dilute magnetic impurities, are a starting point for dealing with a finite density of quantum dots, such as self-assembled quantum dots, or for a finite concentration of magnetic or mixed-valence impurities in bulk [e.g., for $\mathrm{Tl}$ impurities in PbTe (Ref. 9)]. The transport properties of such systems, modeled by a random distribution of Anderson impurities, will be determined by Eqs. (11)-(13) subject to the charge-neutrality condition $n_{i} n_{d}+n_{c}=n$, where $n_{d}$ is the occupancy of the dot (impurity), $n_{c}$ is the occupancy of the relevant conduction band, and $n$ is the total electron filling. Coupled with material-specific 
electronic-structure information and the effects of phonons, such calculations, will be important for understanding the potential of materials such as self-assembled quantum dots or $\mathrm{PbTe}_{1-x} \mathrm{Tl}_{x}$ systems for thermoelectric applications.

\section{ACKNOWLEDGMENTS}

Financial support from the Forschungszentrum Jülich (V.Z.) and supercomputing time from the John von Neumann Institute for Computing (Jülich) is gratefully acknowledged. We thank J. von Delft and A. Weichselbaum for useful comments and discussions.

\section{APPENDIX A: REDUCTION OF TWO-CHANNEL ANDERSON MODEL TO A SINGLE-CHANNEL ANDERSON MODEL}

The reduction in the single-level two-lead Anderson model (1) for a quantum dot, to a single-channel model is, in general, approximate, but as we show here, the approximation is very good (or even exact). One notices first, that the $d$ state of the quantum dot in Eq. (1) only couples to the even combination $t_{L} c_{L k \sigma}+t_{R} c_{R k \sigma}$ of the lead electron states. By using the following canonical transformation:

$$
\begin{aligned}
& t a_{e k \sigma}=t_{L} c_{L k \sigma}+t_{R} c_{R k \sigma}, \\
& t a_{o k \sigma}=t_{L} c_{R k \sigma}-t_{R} c_{L k \sigma},
\end{aligned}
$$

noting that normalization of even/odd states implies $t^{2}=t_{L}^{2}$ $+t_{R}^{2}$, we can rewrite Eq. (1) in terms of even $(e)$ and odd $(o)$ lead states, as follows:

$$
\begin{aligned}
H= & \sum_{k \sigma} \epsilon_{e k \sigma} a_{e k \sigma}^{\dagger} a_{e k \sigma}+\sum_{\sigma} \varepsilon_{d} d_{\sigma}^{\dagger} d_{\sigma}+U n_{d \uparrow} n_{d \downarrow} \\
& +t \sum_{k \sigma}\left(a_{e k \sigma}^{\dagger} d_{\sigma}+\text { H.c. }\right)+H_{o}+H_{\mathrm{pot}} \cdot
\end{aligned}
$$

Here, $\varepsilon_{e k \sigma}=\left(\varepsilon_{L k \sigma} t_{L}^{2}+\varepsilon_{R k \sigma} t_{R}^{2}\right) / t^{2}, H_{o}=\sum_{k \sigma} \epsilon_{o k \sigma} a_{o k \sigma}^{\dagger} a_{o k \sigma}$ is the Hamiltonian for the odd lead electrons with $\varepsilon_{o k \sigma}=\left(\varepsilon_{L k \sigma} t_{R}^{2}\right.$ $\left.+\varepsilon_{R k \sigma} t_{L}^{2}\right) / t^{2}$, and $H_{\mathrm{pot}}=\Sigma_{k \sigma} U_{k}^{e o}\left(a_{e k \sigma}^{\dagger} a_{o k \sigma}+\right.$ H.c. $)$ is a potential scattering term between even and odd lead electrons. Hence, the odd lead electrons do not couple to the dot directly but only indirectly via the potential scattering term. The magnitude of this is given by $U_{k}^{e o}=\left(\varepsilon_{L k \sigma}-\varepsilon_{R k \sigma}\right) t_{L} t_{R} / t^{2}$, which is vanishingly small at low energies. Moreover, it vanishes identically for degenerate leads $\varepsilon_{L k \sigma}=\varepsilon_{R k \sigma}$. The calculations we report in this work, using the single-channel Anderson model

$$
\begin{aligned}
H= & \sum_{k \sigma} \epsilon_{e k \sigma} a_{e k \sigma}^{\dagger} a_{e k \sigma}+\sum_{\sigma} \varepsilon_{d} d_{\sigma}^{\dagger} d_{\sigma}+U n_{d \uparrow} n_{d \downarrow} \\
& +t \sum_{k \sigma}\left(a_{e k \sigma}^{\dagger} d_{\sigma}+\text { H.c. }\right)
\end{aligned}
$$

are therefore a very good approximation, even in general, to those obtained from the two-lead model (1) and identical to those for the case $\varepsilon_{L k \sigma}=\varepsilon_{R k \sigma}$. Since $t^{2}=t_{L}^{2}+t_{R}^{2}$, the hybridization strength $\widetilde{\Gamma}=\pi N_{F} t^{2}$ of the single-channel model is seen to be the relevant single-particle broadening, $\widetilde{\Gamma}_{L}+\widetilde{\Gamma}_{R}$, of the
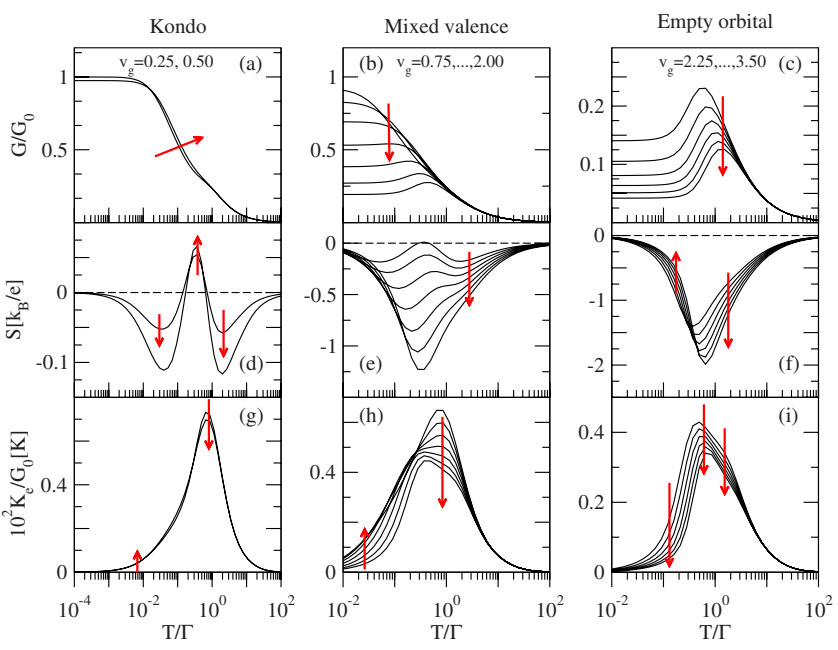

FIG. 11. (Color online) Temperature dependence of, (a)-(c), the normalized electrical conductance $G / G_{0}$, (d)-(f), the thermopower, $S$, and, (g)-(i), the normalized thermal conductance, $K_{\mathrm{e}} / G_{0}$, multiplied by a factor $10^{2}$ for clarity of presentation, as a function of $T / \Gamma$, in the moderately correlated regime $U / \Gamma=3$ and a range of dimensionless gate voltages, $v_{g}=\left(\varepsilon_{d}+U / 2\right) / \Gamma>0$, in the Kondo (first column), mixed-valence (second column), and empty-orbital (third column) regimes. The range of $v_{g}$ is indicated in the top panels for each regime and the increment used was 0.25. Arrows indicate the evolution of the transport quantities with increasing $v_{g}$.

two-lead model (1). In this paper, we follow the convention in the quantum-dot community of using the full width at half maximum, $\Gamma=2 \widetilde{\Gamma}$, as the unit of energy. Finally, we note, that a reduction to a single-channel model is, in general, not possible for multilevel or double quantum dots attached to two leads. ${ }^{73-75}$

\section{APPENDIX B: RESULTS FOR MODERATE AND WEAK CORRELATIONS}

Figure 11 shows results for a moderately correlated quantum dot, $U / \Gamma=3$ exhibiting the same trends as those found for the strongly correlated case $U / \Gamma=8$ (Fig. 3).

For completeness, we show an example of transport through a weakly correlated quantum dot with $U / \Gamma=1$ in Fig. 12. In this case, the thermopower remains negative for all gate voltages $v_{g}>0$ [Fig. 12(b)]. Similarly, the thermal conductance exhibits only a single peak for all gate voltages [Fig. 12(c)].

\section{APPENDIX C: GREEN'S FUNCTIONS WITHIN THE FDM APPROACH}

In this appendix, we give an alternative derivation of the finite-temperature Green's function within the FDM approach of Weichselbaum and von Delft. ${ }^{33}$ A concise derivation, implementing arbitrary abelian symmetries, has also been given in Ref. 35. We consider a general fermionic retarded Green's function,

$$
G_{A B}(t)=-i \theta(t)\left\langle[A(t), B]_{+}\right\rangle=-i \theta(t) \operatorname{Tr}\{\rho[A(t) B+B A(t)]\},
$$

where $A, B$ are fermionic operators, e.g., for the $d$-level Green's function of our quantum $\operatorname{dot} A=d_{\sigma}$ and $B=d_{\sigma}^{\dagger}$. The 


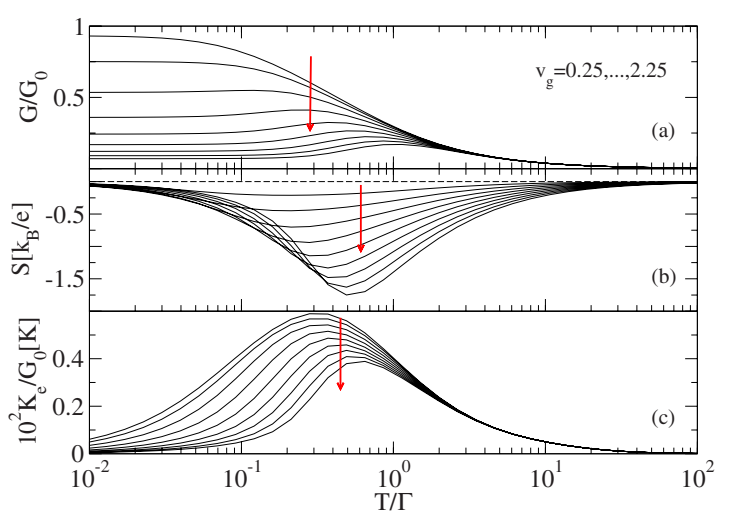

FIG. 12. (Color online) Temperature dependence of, (a), the normalized electrical conductance, $G / G_{0}$, (b), the thermopower, $S$, and, (c), the normalized thermal conductance, $K_{\mathrm{e}} / G_{0}$, multiplied by a factor $10^{2}$ for clarity of presentation, as a function of $T / \Gamma$, in the weakly correlated regime $U / \Gamma=1$ and a range of dimensionless gate voltages, $v_{g}=\left(\varepsilon_{d}+U / 2\right) / \Gamma$. The range of $v_{g}$ is indicated in the top panel and the increment used was 0.25 . Arrows indicate the evolution of the transport quantities with increasing $v_{g}$.

trace is evaluated for an appropriate density matrix $\rho$ by using the complete set of states introduced by Anders and Schiller. ${ }^{37}$ These consist of the set of states $|l e m\rangle=|l m\rangle|e\rangle$ obtained from the eliminated eigenstates, $|l m\rangle$, of $H_{m}$, and the degrees of freedom, denoted collectively by $e$, of the sites $i=m+1, \ldots, N$, where $N$ is the longest chain diagonalized. The retained low energy states of $H_{m}$ are denoted $|k m\rangle$, and $|k e m\rangle=|k m\rangle|e\rangle$ extends these to the Hilbert space of $H_{N}$ by the additional environment degrees of freedom $e$ of the sites $i=m+1, \ldots, N$. The eigenstates (retained and eliminated), $|p=(k, e) m\rangle$, and eigenvalues, $E_{p=(k, l)}^{m}$, of $H_{m}$ satisfy $H_{m}|p m\rangle$ $=E_{p}^{m}|p m\rangle$. Completeness of the states $|l e m\rangle$ is expressed by ${ }^{37}$

$$
1=\sum_{m^{\prime}=m_{0}+1}^{N} \sum_{l e}\left|l e m^{\prime}\right\rangle\left\langle l e m^{\prime}\right|,
$$

where $m_{0}$ is the last iteration for which all states are retained. For iterations $m>m_{0}$, the set of states $|p=(k, l) m\rangle$ consists of both retained $(k)$ and eliminated $(l)$ states. The following decomposition of Eq. (C1) is useful: ${ }^{37}$

$$
\begin{gathered}
\left.1=\sum_{m^{\prime}=m_{0^{+1}}}^{N} \sum_{l e} \mid \text { lem }^{\prime}\right\rangle\left\langle\text { lem }^{\prime}\right|=1_{m}^{+}+1_{m}^{-}, \\
\left.1_{m}^{-}=\sum_{m^{\prime}=m_{0^{+1}}}^{m} \sum_{\text {le }} \mid \text { lem }^{\prime}\right\rangle\left\langle\text { lem }^{\prime}\right|, \\
\left.1_{m}^{+}=\sum_{m^{\prime}=m+1}^{N} \sum_{\text {le }} \mid \text { lem }^{\prime}\right\rangle\left\langle\text { lem }^{\prime}\right| \\
\left.=\sum_{k e} \mid \text { kem }\right\rangle\langle k e m|,
\end{gathered}
$$

where the last equation follows from the fact that the Hilbert space of retained states at iteration $m$ (supplemented by the degrees of freedom $e$ for sites $\left.m^{\prime}=m+1, \ldots, N\right)$ spans the same Hilbert space as all eliminated states from all subsequent iterations. By using the decomposition of unity [Eq. (C1)] twice within the trace in the expression for $G_{A B}(t)$, the following Lehmann representation can be found for this Green's function: ${ }^{34}$

$$
\begin{aligned}
& G_{A B}(t)=G_{A B}^{i}+G_{A B}^{i i}+G_{A B}^{i i i}, \\
& G_{A B}^{i}=-i \theta(t) \sum_{m=m_{0}^{+1}}^{N} \sum_{l e, l^{\prime} e^{\prime}}\left[e^{i\left(E_{l}^{m}-E_{l^{\prime}}^{m}\right) t}\left\langle\text { lem }|A| l^{\prime} e^{\prime} m\right\rangle\right. \\
& \times\left\langle l^{\prime} e^{\prime} m|B \rho| \text { lem }\right\rangle+e^{i\left(E_{l}^{m}-E_{l^{\prime}}^{m}\right) t}\left\langle\text { lem }|A| l^{\prime} e^{\prime} m\right\rangle \\
&\left.\times\left\langle l^{\prime} e^{\prime} m|\rho B| \text { lem }\right\rangle\right], \\
& G_{A B}^{i i}=-i \theta(t) \sum_{m=m_{0}+1}^{N-1} \sum_{l e, k e^{\prime}}\left[e^{i\left(E_{l}^{m}-E_{k}^{m}\right) t}\left\langle k e^{\prime} m|B \rho| \text { lem }\right\rangle\right. \\
& \times\left\langle\text { lem }|A| k e^{\prime} m\right\rangle+e^{i\left(E_{k}^{m}-E_{l}^{m}\right) t}\left\langle\text { lem }|\rho B| k e^{\prime} m\right\rangle \\
&\left.\times\left\langle k e^{\prime} m|A| \text { lem }\right\rangle\right], \\
& G_{A B}^{i i i}=-i \theta(t) \sum_{m=m_{0}+1}^{N-1} \sum_{l e m, k e^{\prime}}\left[e^{i\left(E_{k}^{m}-E_{l}^{m}\right) t}\left\langle\text { lem }|B \rho| k e^{\prime} m\right\rangle\right. \\
& \times\left\langle k e^{\prime} m|A| \text { lem }\right\rangle+e^{i\left(E_{l}^{m}-E_{k}^{m}\right) t}\left\langle k e^{\prime} m|\rho B| \text { lem }\right\rangle \\
&\left.\times\left\langle\text { lem }|A| k e^{\prime} m\right\rangle\right],
\end{aligned}
$$

where the double sum over $m, m^{\prime}$ [coming from two applications of Eq. (C1)] is decomposed into contributions $m^{\prime}$ $=m$ (first term), $m^{\prime}>m$ (second term), and $m^{\prime}<m$ (third term). In the last two terms, use has also been made of Eq. (C4). In the time evolution $e^{i H t} \mid$ pem $\rangle, p=(k, l)$, we have made use of the NRG approximation $H \approx H_{m}$ so that $e^{i H t} \mid$ pem $\rangle \approx e^{i t E_{p}^{m}} \mid$ pem $\rangle$. Peters et al. ${ }^{34}$ evaluated the above expression for the Green's function by using an approximate density matrix $\rho_{N}$, defined by the eliminated states of the longest chain diagonalized, i.e.,

$$
\rho_{N}=\frac{1}{Z_{N}\left(T_{N}\right)} \sum_{l}|l N\rangle e^{-\beta_{N} E_{l}^{N}}\langle l N|,
$$

where $\beta_{N}=1 / k_{B} T_{N}$ (or $T_{N}$ ) is chosen appropriately ${ }^{30}$ to ensure that $Z_{N}\left(T_{N}\right)$ is a good approximation to the partition function of the infinite system at temperature $T=T_{N}$. Note also, that since $N$ is the last iteration, all states in the above expression are considered as eliminated states in order that Eq. (C1) is satisfied. This procedure can be repeated for each chain length $m=N, N-1, \ldots, m_{0}+1$, using a density matrix

$$
\rho_{m}=\frac{1}{Z_{m}\left(T_{m}\right)} \sum_{l}|l m\rangle e^{-\beta_{m} E_{l}^{m}}\langle l m|,
$$

to obtain shell Green's functions $\widetilde{G}_{m}(\omega), m=N, N$ $-1, \ldots, m_{0}+1$ defined at a corresponding set of temperatures $T_{m}$ (or $\beta_{m}=1 / k_{B} T_{m}$ ) (for clarity we henceforth omit the subscript $A B$ for $G_{A B}$ ). Since these shell Green's functions, $\widetilde{G}_{m}$, contain only excitations of order the characteristic scale, $\omega_{m}$, 
of $H_{m}$, or larger, the Green's function $\widetilde{G}_{m}(\omega)$ can only be evaluated at frequencies $\omega>T_{m}$. Information at $\omega \ll T_{m}$ is not available. This restriction is overcome by the FDM approach that we now describe.

Weichselbaum and von Delft ${ }^{33}$ evaluated the above Green's function by using the FDM of the system made up of the complete set of eliminated states from all iterations $m$ $=m_{0}+1, \ldots, N$. Specifically, the FDM is defined by

$$
\rho=\sum_{m=m_{0}+1}^{N} \sum_{l e}|\operatorname{lem}\rangle \frac{e^{-\beta E_{l}^{m}}}{Z(T)}\langle\text { lem }|,
$$

where $Z(T)$ is the partition function made up from the complete spectrum, i.e., it contains all eliminated states from all $H_{m}, m=m_{0}+1, \ldots, N$. Consequently, evaluating the Green's functions by using the above FDM, allows an arbitrary temperature $T$ to be used for all frequencies $\omega$, and, in particular, allows accurate calculations to be carried out at $\omega \ll T$.

Consider the following density matrix for the $m$ th shell (defined, however, in the Hilbert space of $H_{N}$ ):

$$
\tilde{\rho}_{m}=\sum_{l e}|l e m\rangle \frac{e^{-\beta E_{l}^{m}}}{\tilde{Z}_{m}}\langle\text { lem }| .
$$

Normalization, $\operatorname{Tr}\left[\tilde{\rho}_{m}\right]=1$, implies

$$
1=\sum_{l} \frac{e^{-\beta E_{l}^{m}}}{\tilde{Z}_{m}} 4^{N-m}=4^{N-m} \frac{Z_{m}}{\tilde{Z}_{m}},
$$

where $Z_{m}=\Sigma_{l} e^{-\beta E_{l}^{m}}$. Then the FDM can be written as a sum of weighted density matrices for shells $m=m_{0}+1, \ldots, N$,

$$
\begin{gathered}
\rho=\sum_{m=m_{0}+1}^{N} w_{m} \tilde{\rho}_{m}, \\
w_{m}=4^{N-m} \frac{Z_{m}}{Z} ; \sum_{m=m_{0}+1}^{N} w_{m}=1 .
\end{gathered}
$$

The calculation of the weights $w_{m}$ is outlined in the next subsection. Substituting $\rho=\Sigma_{m^{\prime}} w_{m^{\prime}} \widetilde{\rho}_{m^{\prime}}$ into the above Lehmann representation for $G(t)$ and Fourier transforming yields $G(\omega)=\sum_{m^{\prime}} w_{m^{\prime}}\left[G_{m^{\prime}}^{i}(\omega)+G_{m^{\prime}}^{i i}(\omega)+G_{m^{\prime}}^{i i i}(\omega)\right]$. The first term, $G_{m^{\prime}}^{i}$, is easily evaluated by using the orthonormality of the eliminated states $\left\langle l^{\prime} e^{\prime} m^{\prime} \mid l e m\right\rangle=\delta_{l l^{\prime}} \delta_{e e^{\prime}} \delta_{m m^{\prime}}$, orthonormality of environment degrees of freedom in $\left\langle\right.$ lem $\left.|A| l^{\prime} e^{\prime} m^{\prime}\right\rangle$ $=\delta_{e e^{\prime}} A_{l l^{\prime}}^{m^{\prime}}$, with $A_{l l^{\prime}}^{m^{\prime}}=\left\langle l m^{\prime}|A| l^{\prime} m^{\prime}\right\rangle$ and the trace over the $N$ $-m^{\prime}$ environment degrees of freedom in

$$
\frac{1}{\tilde{Z}_{m^{\prime}}} \sum_{e}=\frac{4^{N-m^{\prime}}}{\widetilde{Z}_{m^{\prime}}}=\frac{1}{Z_{m^{\prime}}}
$$

to obtain (for $\left.m^{\prime}=m_{0}+1, \ldots, N\right)$

$$
G_{m^{\prime}}^{i}(\omega)=\frac{1}{Z_{m^{\prime}}} \sum_{l l^{\prime}} A_{l l^{\prime}}^{m^{\prime}} B_{l^{\prime} l}^{m^{\prime} l} \frac{\left(e^{-\beta E_{l}^{m^{\prime}}}+e^{-\beta E_{l^{\prime}}^{m^{\prime}}}\right)}{\omega+E_{l}^{m^{\prime}}-E_{l^{\prime}}^{m^{\prime}}+i \delta} .
$$

The second term, $G_{m^{\prime}}^{i i}$, is also easily evaluated and results for $m^{\prime}=m_{0}+1, \ldots, N-1$ (the $N$ th term vanishes, as all states are counted as eliminated states at this iteration),

$$
\begin{aligned}
G_{m^{\prime}}^{i i}(\omega)= & \frac{1}{Z_{m^{\prime}}} \sum_{l k} A_{l k}^{m^{\prime}} B_{k l}^{m^{\prime}} \frac{e^{-\beta E_{l}^{m^{\prime}}}}{\omega+E_{l}^{m^{\prime}}-E_{k}^{m^{\prime}}+i \delta} \\
& +\frac{1}{Z_{m^{\prime}}} \sum_{k l} A_{k l}^{m^{\prime}} B_{l k}^{m^{\prime}} \frac{e^{-\beta E_{l}^{m^{\prime}}}}{\omega+E_{k}^{m^{\prime}}-E_{l}^{m^{\prime}}+i \delta} .
\end{aligned}
$$

The third term, $G_{m^{\prime}}^{i i i}$, takes the form

$G_{m^{\prime}}^{i i i}(\omega)=\sum_{l e k} A_{l k}^{m} \frac{\left\langle k e m\left|\tilde{\rho}_{m^{\prime}} B\right| l e m\right\rangle}{\omega+E_{l}^{m}-E_{k}^{m}+i \delta}+\sum_{k l e} A_{k l}^{m} \frac{\left\langle l e m\left|B \tilde{\rho}_{m^{\prime}}\right| k e m\right\rangle}{\omega+E_{k}^{m}-E_{l}^{m}+i \delta}$.

Inserting $1=1_{m}^{+}+1_{m}^{-}$between $\widetilde{\rho}_{m^{\prime}}$ and $B$ in $\left\langle\right.$ kem $\left|\widetilde{\rho}_{m^{\prime}} B\right|$ lem $\rangle$ and between $B$ and $\widetilde{\rho}_{m^{\prime}}$ in $\left\langle\right.$ lem $\left|B \widetilde{\rho}_{m^{\prime}}\right|$ kem $\rangle$ gives

$$
\left\langle\text { kem }\left|\widetilde{\rho}_{m^{\prime}} B\right| \text { lem }\right\rangle=\left\langle\text { kem }\left|1_{m}^{+} \widetilde{\rho}_{m^{\prime}} B\right| \text { lem }\right\rangle+\left\langle\text { kem }\left|1_{m}^{-} \widetilde{\rho}_{m^{\prime}} B\right| \text { lem }\right\rangle
$$

and

$$
\left\langle\text { lem }\left|B \widetilde{\rho}_{m^{\prime}}\right| k e m\right\rangle=\left\langle\operatorname{lem}\left|B 1_{m}^{+} \widetilde{\rho}_{m^{\prime}}\right| k e m\right\rangle+\left\langle l e m\left|B 1_{m}^{-} \widetilde{\rho}_{m^{\prime}}\right| k e m\right\rangle
$$

In the last subsection, we show that the second terms in the above expressions vanish, i.e.,

$$
\left\langle\text { kem }\left|1_{m}^{-} \tilde{\rho}_{m^{\prime}} B\right| \text { lem }\right\rangle=0,
$$

$$
\left\langle\text { lem }\left|B 1_{m}^{-} \tilde{\rho}_{m^{\prime}}\right| k e m\right\rangle=0 .
$$

On using $1_{m}^{+}=\Sigma_{k^{\prime} e^{\prime}}\left|k^{\prime} e^{\prime} m\right\rangle\left\langle k^{\prime} e^{\prime} m\right|$ from Eq. (C4) the terms involving $1_{m}^{+}$are evaluated as

$$
\begin{aligned}
\left\langle\text { kem }\left|1_{m}^{+} \tilde{\rho}_{m^{\prime}} B\right| \text { lem }\right\rangle & =\sum_{k^{\prime} e^{\prime}}\left\langle\text { kem }\left|\tilde{\rho}_{m^{\prime}}\right| k^{\prime} e^{\prime} m\right\rangle\left\langle k^{\prime} e^{\prime} m|B| \text { lem }\right\rangle \\
& =\sum_{k^{\prime}}\left\langle\text { kem }\left|\widetilde{\rho}_{m^{\prime}}\right| k^{\prime} \text { em }\right\rangle\left\langle k^{\prime} \text { em }|B| \text { lem }\right\rangle \\
& =\sum_{k^{\prime}}\left\langle\text { kem }\left|\widetilde{\rho}_{m^{\prime}}\right| k^{\prime} \text { em }\right\rangle B_{k^{\prime} l}^{m}, \\
\left\langle\text { lem }\left|B 1_{m}^{+} \widetilde{\rho}_{m^{\prime}}\right| \text { kem }\right\rangle & =\sum_{k^{\prime} e^{\prime}}\left\langle\text { lem }|B| k^{\prime} e^{\prime} m\right\rangle\left\langle k^{\prime} e^{\prime} m\left|\widetilde{\rho}_{m^{\prime}}\right| k e m\right\rangle \\
& =\sum_{k^{\prime}}\left\langle\text { lem }|B| k^{\prime} \text { em }\right\rangle\left\langle k^{\prime} \text { em }\left|\widetilde{\rho}_{m^{\prime}}\right| k e m\right\rangle \\
& =\sum_{k^{\prime}} B_{l k^{\prime}}^{m}\left\langle k^{\prime} \text { em }\left|\widetilde{\rho}_{m^{\prime}}\right| k e m\right\rangle .
\end{aligned}
$$

Note that these expressions are finite only for $m^{\prime}>m$. Using the definition of the reduced density matrix, ${ }^{36}$

$$
\rho_{\text {red }}^{m^{\prime} \rightarrow m}\left(k, k^{\prime}\right)=\operatorname{Tr}_{\mathrm{e}}\left[\left\langle k e m\left|\widetilde{\rho}_{m^{\prime}}\right| k^{\prime} e m\right\rangle\right],
$$

we arrive at the following expression for $G_{m^{\prime}}^{i i i}(\omega)$ : 


$$
\begin{aligned}
G_{m^{\prime}}^{i i i}(\omega)= & \sum_{m=m_{0}+1}^{m^{\prime}-1} \sum_{l k k^{\prime}} A_{l k}^{m} \frac{\rho_{\mathrm{red}}^{m^{\prime} \rightarrow m}\left(k, k^{\prime}\right) B_{k^{\prime} l}^{m}}{\omega+E_{l}^{m}-E_{k}^{m}+i \delta} \\
& +\sum_{m=m_{0}+1}^{m^{\prime}-1} \sum_{l k k^{\prime}} A_{k l}^{m} \frac{\rho_{\mathrm{red}}^{m^{\prime} \rightarrow m}\left(k^{\prime}, k\right) B_{l k^{\prime}}^{m}}{\omega+E_{k}^{m}-E_{l}^{m}+i \delta} .
\end{aligned}
$$

Hence, the final expression for $G(\omega)=\sum_{m^{\prime}=m_{0}+1}^{N} w_{m^{\prime}}\left(G_{m^{\prime}}^{i}(\omega)\right.$ $\left.+G_{m^{\prime}}^{i i}(\omega)+G_{m^{\prime}}^{i i i}(\omega)\right)$ is given by

$$
\begin{aligned}
G(\omega)= & \sum_{m^{\prime}=m_{0}+1}^{N} \frac{w_{m^{\prime}}}{Z_{m^{\prime}}} \sum_{l l^{\prime}} A_{l l^{\prime}}^{m^{\prime}} B_{l^{\prime} l}^{m^{\prime} l} \frac{\left(e^{-\beta E_{l}^{m^{\prime}}}+e^{-\beta E_{l^{\prime}}^{m^{\prime}}}\right)}{\omega+E_{l}^{m^{\prime}}-E_{l^{\prime}}^{m^{\prime}}+i \delta} \\
& +\sum_{m^{\prime}=m_{0}+1}^{N-1} \frac{w_{m^{\prime}}}{Z_{m^{\prime}}} \sum_{l k} A_{l k}^{m^{\prime}} B_{k l}^{m^{\prime}} \frac{e^{-\beta E_{l}^{m^{\prime}}}}{\omega+E_{l}^{m^{\prime}}-E_{k}^{m^{\prime}}+i \delta} \\
& +\sum_{m^{\prime}=m_{0}+1}^{N-1} \frac{w_{m^{\prime}}}{Z_{m^{\prime}}} \sum_{k l} A_{k l}^{m^{\prime}} B_{l k}^{m^{\prime}} \frac{e^{-\beta E_{l}^{m^{\prime}}}}{\omega+E_{k}^{m^{\prime}}-E_{l}^{m^{\prime}}+i \delta} \\
& +\sum_{m=m_{0}+1}^{N-1} \sum_{l k k^{\prime}} A_{l k}^{m} \frac{\mathrm{R}_{\mathrm{red}}^{m}\left(k, k^{\prime}\right) B_{k^{\prime} l}^{m}}{\omega+E_{l}^{m}-E_{k}^{m}+i \delta} \\
& +\sum_{m=m_{0}+1}^{N-1} \sum_{k k^{\prime} l} A_{k l}^{m} \frac{\mathrm{R}_{\mathrm{red}}^{m}\left(k^{\prime}, k\right) B_{l k^{\prime}}^{m}}{\omega+E_{k}^{m}-E_{l}^{m}+i \delta},
\end{aligned}
$$

where in the last two terms, we rearranged the summations over $m^{\prime}$ and $m$ and introduced the full reduced density matrix,

$$
\mathrm{R}_{\mathrm{red}}^{m}\left(k, k^{\prime}\right)=\sum_{m^{\prime}=m+1}^{N} w_{m^{\prime}} \rho_{\mathrm{red}}^{m^{\prime} \rightarrow m}\left(k, k^{\prime}\right) .
$$

Note that the meaning of this quantity is completely analogous to the reduced density matrix introduced by Hofstetter in Ref. 36 except that one obtains reduced density matrices at iteration $m$ by eliminating environment degrees of freedom $e=e_{m+1} e_{m+2} \ldots e_{N}$ from the FDM [Eq. (C7)] instead of the density matrix for iteration $N$. In addition, the former is built from the complete set of eliminated states, as opposed to the retained states of iteration $N$ in the approach of Ref. 36. The above expression for $G(\omega)$ is identical to that in Ref. 33. We have checked that the sum rule for the spectral function $A_{\sigma}(\omega, T)=-\frac{1}{\pi} \operatorname{Im}\left[G_{A B}(\omega)\right]$,

$$
\int_{-\infty}^{+\infty} A_{\sigma}(\omega, T) d \omega=1
$$

is satisfied exactly (to machine precision) when using the discrete (unbroadened) form of the spectral function as in Ref. 33.

\section{Calculation of weights $w_{m}$}

The expression for $w_{m}$ in Eq. (C11) involves $Z$ which contains eigenvalues from all iterations $m^{\prime}=m_{0}+1, \ldots, N$. In evaluating these expressions, one should therefore use the absolute energies for the $E_{l}^{m}$. Since, in practice, the iterative diagonalization of the Hamiltonian $H_{m}$ involves subtraction of ground-state energies and rescaling at each $m$ (see Ref. 30 ), one has to keep track of the subtracted ground-state energies and return to the actual physical energies relative to a common absolute energy reference in evaluating $w_{m}$ and $Z$. We take this absolute energy reference to be the ground-state energy of the last Wilson iteration $N$. Thus, if $E_{G S}^{m}$ is the true ground-state energy of $H_{m}$, we use $E_{l}^{m} \rightarrow E_{l}^{m}+E_{G S}^{m}$ and $Z_{m}$ $\rightarrow e^{-\beta E_{G S}^{m}} Z_{m}$ in evaluating $w_{m} / Z_{m}$ and $Z$,

$$
\begin{aligned}
\frac{w_{m}}{Z_{m}} & =\frac{4^{N-m} e^{-\beta E_{G S}^{m}}}{\sum_{m^{\prime}=m_{0}+1}^{N} 4^{N-m^{\prime}} e^{-\beta E_{G S}^{m^{\prime}} Z_{m^{\prime}}}}, \\
Z & =\sum_{m^{\prime}=m_{0^{+1}}}^{N} 4^{N-m^{\prime}} e^{-\beta E_{G S}^{m^{\prime}} Z_{m^{\prime}}}
\end{aligned}
$$

\section{Proof of Eqs. (C12) and (C13)}

Using the expression for $\tilde{\rho}_{m^{\prime}}$, we easily find that

$$
\begin{aligned}
1_{m}^{-} \tilde{\rho}_{m^{\prime}}= & \left.\sum_{m^{\prime \prime}=m_{0}+1}^{m} \sum_{l e} \mid \text { lem }{ }^{\prime \prime}\right\rangle\left\langle\text { lem }{ }^{\prime \prime}\right| \\
& \times \sum_{l^{\prime} e^{\prime}}\left|l^{\prime} e^{\prime} m^{\prime}\right\rangle \frac{e^{-\beta E_{l^{\prime}}^{m^{\prime}}}}{\tilde{Z}_{m^{\prime}}}\left\langle l^{\prime} e^{\prime} m^{\prime}\right| \\
= & \sum_{m^{\prime \prime}=m_{0}+1}^{m} \delta_{m^{\prime \prime} m^{\prime}} \sum_{l e}\left|l e m^{\prime}\right\rangle \frac{e^{-\beta E_{l^{\prime}}^{m^{\prime}}}}{\tilde{Z}_{m^{\prime}}}\langle\text { lem }| \\
= & \begin{cases}\tilde{\rho}_{m^{\prime}} & \text { if } m^{\prime} \leq m ; \\
0 & \text { if } m^{\prime}>m .\end{cases}
\end{aligned}
$$

Hence $\left\langle k e m\left|1_{m}^{-} \widetilde{\rho}_{m^{\prime}} B\right|\right.$ lem $\rangle$ in Eq. (C12) involves matrix elements of the form $\left\langle k e m \mid l^{\prime} e^{\prime} m^{\prime}\right\rangle$ for $m^{\prime} \leq m$, which vanish, since all retained state at iteration $m$ have no overlap with eliminated states at iterations $m^{\prime} \leq m$ (i.e., eliminated states of previous iterations are not used to obtain retained states of later iterations). The same arguments can be used to prove Eq. (C13).

\section{APPENDIX D: THERMAL CONDUCTANCE AND THERMOPOWER OF QUANTUM DOTS}

For completeness, we outline here the derivation of thermoelectric transport through a strongly interacting quantum dot. $^{21,22}$ The electrical, $I_{L}$, and heat current, $I_{L}^{Q}$, from the left lead to the quantum dot can be expressed in terms of the particle number $N_{L}=\Sigma_{k \sigma} c_{L k \sigma}^{\dagger} c_{L k \sigma}$ and energy $H_{L}$ $=\Sigma_{k \sigma} \epsilon_{L k \sigma} c_{L k \sigma}^{\dagger} c_{L k \sigma}$ of the left lead, via

$$
I_{L}=-e \dot{N}_{L}=-\frac{e}{i \hbar}\left[N_{L}, H\right]
$$




$$
I_{L}^{Q}=\dot{H}_{L}-\mu_{L} \dot{N}_{L}=\frac{1}{i \hbar}\left[H_{L}-\mu_{L} N_{L}, H\right],
$$

where $H$ is the Hamiltonian (1). In terms of the lesser Green's function's $G_{d \sigma, k L \sigma}^{<}\left(t, t^{\prime}\right)=i\left\langle c_{k L \sigma}^{\dagger}\left(t^{\prime}\right) d_{\sigma}(t)\right\rangle \quad$ and $G_{k L \sigma, d \sigma}^{<}\left(t, t^{\prime}\right)=i\left\langle d_{\sigma}^{\dagger}\left(t^{\prime}\right) c_{k L \sigma}(t)\right\rangle=-\left(G_{d \sigma, k L \sigma}^{<}\right)^{*}$, the above currents are given by

$$
\begin{gathered}
I_{L}=\frac{2 e}{\hbar} \operatorname{Re}\left[\sum_{k \sigma} t_{L} G_{d \sigma, k L \sigma}^{<}(t, t)\right], \\
I_{L}^{Q}=-\frac{2}{\hbar} \operatorname{Re}\left[\sum_{k \sigma} t_{L}\left(\varepsilon_{L k \sigma}-\mu_{L}\right) G_{d \sigma, k L \sigma}^{<}(t, t)\right] .
\end{gathered}
$$

The lesser Green's function $G_{d \sigma, k L \sigma}^{<}\left(t, t^{\prime}\right)$ can be expressed via equations of motion solely in terms of Green's functions of the dot and the noninteracting Green's function for the left lead. After some lengthy algebra, ${ }^{22,40}$ one finds the following expressions for the currents in terms of the retarded, $G_{d \sigma}^{r}$ $=G_{d \sigma}(\omega+i \delta)$, advanced, $G_{d \sigma}^{a}=G_{d \sigma}(\omega-i \delta)$ and lesser Green's function, $G_{d \sigma}^{<}(\omega)$ of the dot,

$$
\begin{gathered}
I_{L}=\frac{i e}{\hbar} \sum_{\sigma} \int d \omega \widetilde{\Gamma}_{L}\left[\left(G_{d \sigma}^{<}(\omega)+f_{L}(\omega)\left(G_{d \sigma}^{r}-G_{d \sigma}^{a}\right)\right], \quad(\mathrm{D} 5)\right. \\
I_{L}^{Q}=-\frac{i}{\hbar} \sum_{\sigma} \int d \omega\left(\omega-\mu_{L}\right) \widetilde{\Gamma}_{L}\left[G_{d \sigma}^{<}(\omega)+f_{L}(\omega)\left(G_{d \sigma}^{r}-G_{d \sigma}^{a}\right)\right],
\end{gathered}
$$

where $f_{L}(\omega)=\left(1+e^{-\left(\omega-\mu_{L}\right) /\left(k_{B} T_{L}\right)}\right)^{-1}$ is the Fermi function of the left lead and $\widetilde{\Gamma}_{L}=\pi N_{F} t_{L}^{2}$ is the hybridization strength of the dot to the left lead as defined in Sec. II. By using current conservation $I_{L}=-I_{R}$, one can eliminate the lesser Green's function from the above expressions to arrive at the final expressions used in this paper,

$$
\begin{gathered}
I_{L}=\frac{e}{\hbar} \sum_{\sigma} \int d \omega\left(f_{L}-f_{R}\right) \mathcal{T}_{d \sigma}(\omega), \\
I_{L}^{Q}=-\frac{i}{\hbar} \sum_{\sigma} \int d \omega\left(\omega-\mu_{L}\right)\left(f_{L}-f_{R}\right) \mathcal{T}_{d \sigma}(\omega) .
\end{gathered}
$$

The quantity $\mathcal{T}_{d \sigma}(\omega)$ acts as a transmission function and is given by

$$
\mathcal{T}_{d \sigma}(\omega)=2 i \frac{\widetilde{\Gamma}_{L} \tilde{\Gamma}_{R}}{\widetilde{\Gamma}_{L}+\widetilde{\Gamma}_{R}}\left(G_{d \sigma}^{r}-G_{d \sigma}^{a}\right) .
$$

The electric and heat currents are expanded to linear order in $\delta T=T_{L}-T_{R}$ and $\delta V=V_{L}-V_{R}$,

$$
\left(\begin{array}{c}
I_{L} \\
I_{L}^{Q}
\end{array}\right)=\left(\begin{array}{ll}
L_{11} & L_{12} \\
L_{21} & L_{22}
\end{array}\right)\left(\begin{array}{c}
\delta V \\
\delta T
\end{array}\right),
$$

defining, thereby, the transport coefficients $L_{i, j}, i, j=1,2$. In terms of the latter, the transport properties are given by

$$
\begin{gathered}
G(T)=\lim _{\delta V \rightarrow 0} I_{L} /\left.\delta V\right|_{\delta T=0}=L_{11}, \\
S(T)=-\lim _{\delta T \rightarrow 0} \delta V /\left.\delta T\right|_{I_{L}=0}=L_{12} / L_{11}, \\
K_{\mathrm{e}}(T)=-\lim _{\delta T \rightarrow 0} I^{Q} /\left.\delta T\right|_{I_{L}=0}=L_{12} L_{21} / L_{11}-L_{22} .
\end{gathered}
$$

Finally, the $L_{i j}$ are simply expressed in terms of the following transport integrals:

$$
I_{n}(T)=\frac{2}{h} \int d \epsilon \epsilon^{n} T(\epsilon)\left[-\frac{\partial f}{\partial \varepsilon}\right],
$$

via $L_{11}=e^{2} I_{0}, L_{21}=-e I_{1} / T$ and $L_{22}=I_{2} / T$. Substituting these values for $L_{i j}$ into Eqs. (D11)-(D13) results in the expressions (4)-(6) given in the text.
${ }^{1}$ G. D. Mahan, Solid State Phys. 51, 81 (1998).

${ }^{2}$ M. G. Kanatzidis, Chem. Mater. 22, 648 (2010).

${ }^{3}$ I. Terasaki, Y. Sasago, and K. Uchinokura, Phys. Rev. B 56, R12685 (1997).

${ }^{4}$ R. Arita, K. Kuroki, K. Held, A. V. Lukoyanov, S. Skornyakov, and V. I. Anisimov, Phys. Rev. B 78, 115121 (2008).

${ }^{5}$ R. Lackner, E. Bauer, and P. Rogl, Physica B 378-390, 835 (2006).

${ }^{6} \mathrm{~S}$. Paschen, in Thermoelectric Handbook, edited by D. M. Rowe (CRC Press, Taylor \& Francis, Boca Raton, FL, 2006).

${ }^{7}$ A. Bentien, S. Johnsen, G. K. H. Madsen, B. B. Iversen, and F. Steglich, EPL 80, 17008 (2007).

${ }^{8}$ B. C. Sales, D. Mandrus, and R. K. Williams, Science 272, 1325 (1996); R. P. Hermann, R. Jin, W. Schweika, F. Grandjean, D. Mandrus, B. C. Sales, and G. J. Long, Phys. Rev. Lett. 90, 135505 (2003).

${ }^{9}$ M. Matusiak, E. M. Tunnicliffe, J. R. Cooper, Y. Matsushita, and I. R. Fisher, Phys. Rev. B 80, 220403(R) (2009).
${ }^{10}$ K. F. Hsu, S. Loo, F. Guo, W. Chen, J. S. Dyck, C. Uher, T. Hogan, E. K. Polychroniadis, and M. Kanatzidis, Science 303, 818 (2004).

${ }^{11}$ R. Venkatasubramanian, E. Siivola, T. Colpitts, and B. O’Quinn, Nature (London) 413, 597 (2001).

${ }^{12}$ J. Cai and G. D. Mahan, Phys. Rev. B 78, 035115 (2008).

${ }^{13}$ T. C. Harman, P. J. Taylor, M. P. Walsh, and B. E. Laforge, Science 297, 2229 (2002).

${ }^{14}$ H. Beyer, J. Nurnus, H. Böttner, A. Lambrecht, T. Roch, and G. Bauer, Appl. Phys. Lett. 80, 1216 (2002).

${ }^{15}$ T. A. Costi, A. C. Hewson, and V. Zlatić, J. Phys.: Condens. Matter 6, 2519 (1994); T. A. Costi and A. C. Hewson, ibid. 5, L361 (1993).

${ }^{16}$ V. Zlatić, T. A. Costi, A. C. Hewson, and B. R. Coles, Phys. Rev. B 48, 16152 (1993).

${ }^{17}$ N. E. Bickers, D. L. Cox, and J. W. Wilkins, Phys. Rev. B 36, 2036 (1987); Phys. Rev. Lett. 54, 230 (1985); N. E. Bickers, Rev. Mod. Phys. 59, 845 (1987). 
${ }^{18}$ V. Zlatić and R. Monnier, Phys. Rev. B 71, 165109 (2005).

${ }^{19}$ C. Grenzenbach, F. B. Anders, and G. Czycholl, in Properties and Applications of Thermoelectric Materials, NATO Advanced Research Workshop, Series B: Physics and Biophysics, edited by V. Zlatić and A. C. Hewson, (Springer, Dordrecht, 2009).

${ }^{20}$ R. Scheibner, H. Buhmann, D. Reuter, M. N. Kiselev, and L. W. Molenkamp, Phys. Rev. Lett. 95, 176602 (2005).

${ }^{21}$ B. Dong and X. L. Lei, J. Phys.: Condens. Matter 14, 11747 (2002).

${ }^{22}$ T.-S. Kim and S. Hershfield, Phys. Rev. Lett. 88, 136601 (2002); Phys. Rev. B 67, 165313 (2003).

${ }^{23}$ D. Goldhaber-Gordon, J. Göres, M. A. Kastner, H. Shtrikman, D. Mahalu, and U. Meirav, Phys. Rev. Lett. 81, 5225 (1998).

${ }^{24}$ J. Schmid, J. Weis, K. Eberl, and K. v. Klitzing, Physica B 256258, 182 (1998); Phys. Rev. Lett. 84, 5824 (2000).

${ }^{25}$ S. M. Cronenwett, T. H. Osterkamp, and L. P. Kouwenhoven, Science 281, 540 (1998).

${ }^{26}$ W. van der Wiel, S. De Francheschi, T. Fujisawa, J. M. Elzerman, S. Tarucha, and L. P. Kouwenhoven, Science 289, 2105 (2000).

${ }^{27}$ J. Nygard, D. H. Cobden, and P. E. Lindelof, Nature (London) 408, 342 (2000).

${ }^{28}$ R. Scheibner, E. G. Novik, T. Borzenko, M. König, D. Reuter, A. D. Wieck, H. Buhmann, and L. W. Molenkamp, Phys. Rev. B 75, 041301(R) (2007).

${ }^{29}$ K. G. Wilson, Rev. Mod. Phys. 47, 773 (1975).

${ }^{30}$ H. R. Krishna-murthy, J. W. Wilkins, and K. G. Wilson, Phys. Rev. B 21, 1003 (1980).

${ }^{31}$ R. Bulla, T. A. Costi, and T. Pruschke, Rev. Mod. Phys. 80, 395 (2008).

${ }^{32}$ R. Bulla, A. C. Hewson, and Th. Pruschke, J. Phys.: Condens. Matter 10, 8365 (1998).

${ }^{33}$ A. Weichselbaum and J. von Delft, Phys. Rev. Lett. 99, 076402 (2007).

${ }^{34}$ R. Peters, T. Pruschke, and F. B. Anders, Phys. Rev. B 74, 245114 (2006).

${ }^{35}$ A. I. Tóth, C. P. Moca, Ö. Legeza, and G. Zaránd, Phys. Rev. B 78, 245109 (2008).

${ }^{36}$ W. Hofstetter, Phys. Rev. Lett. 85, 1508 (2000).

${ }^{37}$ F. B. Anders and A. Schiller, Phys. Rev. Lett. 95, 196801 (2005).

${ }^{38}$ Y. Meir, N. S. Wingreen, and P. A. Lee, Phys. Rev. Lett. 70, 2601 (1993).

${ }^{39}$ S. Hershfield, J. H. Davies, and J. W. Wilkins, Phys. Rev. Lett. 67, 3720 (1991); Phys. Rev. B 46, 7046 (1992).

${ }^{40}$ A. P. Jauho, N. S. Wingreen, and Y. Meir, Phys. Rev. B 50, 5528 (1994).

${ }^{41}$ T. A. Costi, Phys. Rev. B 55, 3003 (1997).

${ }^{42}$ A. C. Hewson, The Kondo Problem To Heavy Fermions, Cambridge Studies in Magnetism (Cambridge University Press, Cambridge, England, 1997).

${ }^{43}$ L. I. Glazman and M. E. Raikh, JETP Lett. 47, 452 (1988).

${ }^{44}$ T.-K. Ng and P. A. Lee, Phys. Rev. Lett. 61, 1768 (1988).

${ }^{45}$ T. A. Costi, Phys. Rev. B 64, 241310(R) (2001).

${ }^{46}$ W. Izumida, O. Sakai, and S. Suzuki, J. Phys. Soc. Jpn. 70, 1045 (2001); O. Sakai, S. Suzuki, W. Izumida, and A. Oguri, ibid. 68, 1640 (1999).

${ }^{47}$ T. A. Costi, Phys. Rev. Lett. 85, 1504 (2000).

${ }^{48}$ T. A. Costi, in Concepts in Electron Correlation, edited by V.
Zlatić and A. C. Hewson (Springer, Dordrecht, 2003), p. 247.

${ }^{49}$ H. Schoeller and J. König, Phys. Rev. Lett. 84, 3686 (2000).

${ }^{50}$ L. Craco and K. Kang, Phys. Rev. B 59, 12244 (1999).

${ }^{51}$ A. Martin-Rodero, F. Flores, M. Baldo, and R. Pucci, Solid State Commun. 44, 911 (1982).

${ }^{52}$ M. Yoshida and L. N. Oliveira, Physica B 404, 3312 (2009).

${ }^{53}$ D. Boese and R. Fazio, Europhys. Lett. 56, 576 (2001).

${ }^{54}$ R. Franco, J. Silva-Valencia, and M. S. Figueira, J. Magn. Magn. Mater. 320, e242 (2008).

${ }^{55}$ D. Vollhardt, Phys. Rev. Lett. 78, 1307 (1997).

${ }^{56}$ T. A. Costi and G. Zaránd, Phys. Rev. B 59, 12398 (1999).

${ }^{57}$ M. S. Laad, L. Craco, and E. Müller-Hartmann, Phys. Rev. B 64, 075108 (2001).

${ }^{58}$ B. Kubala, J. König, and J. Pekola, Phys. Rev. Lett. 100, 066801 (2008); A. Garg, D. Rasch, E. Shimshoni, and A. Rosch, ibid. 103, 096402 (2009); R. Świrkowicz, M. Wierzbicki, and J. Barnaś, Phys. Rev. B 80, 195409 (2009).

${ }^{59}$ The quantity $\gamma \sim 1 / T_{K}$ is the linear coefficient of specific heat of the quantum dot. The specific heat, $C(T)$, of a quantum dot is difficult to measure, but theoretically it is easily calculated from the free energy (Ref. 30). The linear coefficient of specific heat, $\gamma$, is then obtained via $\gamma=\lim _{T \rightarrow 0} C(T) / T$.

${ }^{60}$ X. Zianni, Phys. Rev. B 75, 045344 (2007).

${ }^{61}$ M. Tsaousidou and G. P. Triberis, Physics of Semiconductors: 28th International Conference on the Physics of Semiconductors ICPS 2006, AIP Conf. Proc. Vol. 893 (AIP, New York, 2007), pp. 801-802.

${ }^{62}$ C. W. J. Beenakker and A. A. M. Staring, Phys. Rev. B 46, 9667 (1992).

${ }^{63}$ A. A. M. Staring, L. W. Molenkamp, B. W. Alpenhaar, H. van Houten, O. J. A. Buijk, M. A. A. Mabesoone, C. W. J. Beenakker, and C. T. Foxon, Europhys. Lett. 22, 57 (1993).

${ }^{64}$ H. van Houten, L. W. Molenkamp, C. W. J. Beenakker, and C. T. Foxon, Semicond. Sci. Technol. 7, B215 (1992).

${ }^{65}$ L. W. Molenkamp, Th. Gravier, H. van Houten, O. J. A. Buijk, M. A. A. Mabesoone, and C. T. Foxon, Phys. Rev. Lett. 68, 3765 (1992).

${ }^{66}$ A. S. Dzurak, C. G. Smith, M. Pepper, D. A. Ritchie, J. E. F. Frost, G. A. C. Jones, and D. G. Hasko, Solid State Commun. 87, 1145 (1993).

${ }^{67}$ D. H. Cobden, A. S. Dzurak, M. Field, C. G. Smith, A. K. Savchenko, M. Pepper, D. A. Ritchie, J. E. F. Frost, G. A. C. Jones, and D. G. Hasko, Physica A 200, 65 (1993).

${ }^{68}$ A. S. Dzurak, C. G. Smith, C. H. W. Barnes, M. Pepper, L. Martín-Moreno, C. T. Liang, D. A. Ritchie, and G. A. C. Jones, Phys. Rev. B 55, R10197 (1997).

${ }^{69}$ S. Möller, H. Buhmann, S. F. Godijn, and L. W. Molenkamp, Phys. Rev. Lett. 81, 5197 (1998).

${ }^{70}$ A. V. Andreev and K. A. Matveev, Phys. Rev. Lett. 86, 280 (2001); K. A. Matveev and A. V. Andreev, Phys. Rev. B 66, 045301 (2002)

${ }^{71}$ M. Turek and K. A. Matveev, Phys. Rev. B 65, 115332 (2002).

${ }^{72}$ T. K. T. Nguyen, M. N. Kiselev, and V. E. Kravtsov, arXiv:0912.4632 (unpublished).

${ }^{73}$ S. Y. Cho and R. H. McKenzie, Phys. Rev. B 71, 045317 (2005).

${ }^{74}$ R. Sakano, T. Kita, and N. Kawakami, J. Phys. Soc. Jpn. 76, 074709 (2007).

${ }^{75}$ T. Nakanishi and T. Kato, J. Phys. Soc. Jpn. 76, 034715 (2007). 